\title{
Inclusive selection of intermediate-mass-fragment formation modes in the spallation of ${ }^{136} \mathrm{Xe}$
}

\author{
P.Napolitani ${ }^{1,2}$, K.-H.Schmidt ${ }^{2}$ and L.Tassan-Got ${ }^{1}$ \\ ${ }^{1}$ IPN Orsay, Université Paris-Sud 11, CNRS/IN2P3, 91406 Orsay cedex, France \\ ${ }^{2}$ GSI, Planckstr. 1, 64291 Darmstadt, Germany \\ E-mail: napolita@ipno.in2p3.fr
}

\begin{abstract}
A correlation between the production and kinematic properties of the fragments issued of fission and multifragmentation is established in the study of the reaction ${ }^{136} \mathrm{Xe}+$ hydrogen at $1 \mathrm{GeV}$ per nucleon, measured in inverse kinematics at the FRagment Separator (GSI, Darmstadt). Such observables are analysed in a comprehensive study, selected as a function of the decay mode, and related to the isotopic properties of the fragments in the intermediate-mass region. Valuable information can be deduced on the characteristics of the heaviest product in the reaction, which has been considered a fundamental observable for tagging the thermodynamic properties of finite nuclear systems.
\end{abstract}

PACS numbers: 25.40.Sc, 25.70.Pq, 24.60.Dr, 29.30.Aj, 29.87.+g

Keywords: Longitudinal-velocity spectra, isotopic cross sections, intermediate-mass fragments, inclusive isotopic identification 


\section{Introduction}

We present a comprehensive experimental study of the decay processes occurring in the spallation of ${ }^{136} \mathrm{Xe}$ induced by $1 \mathrm{GeV}$ protons, through inclusive kinematic and production observables. The incident energy, the size and the neutron enrichment of the system we chose is compatible with the threshold for multifragmentation. This situation is expected to be characterised by fluctuations between asymmetric fission and multifragmentation. Such scenario has already been presented in a previous work [1], where cross sections were published for the whole production distribution, without any selection on the reaction mechanism. In this work we develop a selection method to identify and quantify the relative shares of the decay processes, fission and multifragmentation, which lead to the production of intermediate-mass fragments. Once established a correlation between production and kinematic quantities as a function of the isotopic observable and the decay mode, we analysed the characteristics of the intermediate-mass fragments issued of fission and multifragmentation, with the further aim of determining a set of observables relevant for describing the decay phenomenology.

\subsection{Context}

In relativistic nuclear reactions, especially when induced by hadrons or light nuclei, kinematic and thermal observables probe the transition between different reaction mechanisms as a function of the bombarding energy [2, 3]. Already some decades ago, several experiments were focused on spallation with the purpose of studying the reaction mechanism (for a non-exhaustive series of pioneering experiments see refs. 4, 5, 6, 7, 8, 9, 10, 11, 12]). In nucleon-induced reactions, at the incident energy of few hundred $\mathrm{MeV}$ per nucleon, an excited and fully equilibrated compound nucleus [13] is formed, which decays by the emission of fragments in the form of particle evaporation and fission, according to the number of final states available for different mass splits [14, 15]. At the incident energy of several GeV per nucleon, the highly excited composite nuclear system has also a large probability of decaying by multifragmentation. For a comprehensive review on this subject see ref. [16]; see also ref. [17] for a large collection of reviews on this process, and refs. [18, 19, 20] for specific reviews treating multifragmentation induced by high-energy protons. In nucleon-induced reactions at incident energies which are intermediate between these extreme situations, for instance around $1 \mathrm{GeV}$ per nucleon, the multifragmentation threshold could be attained: in this case, the exit channel of the system is determined by the competition between multifragmentation and sequential decay.

Such a complex phenomenology inspired the formulation of several alternative descriptions, attempted since the early eighties, in order to understand the transition between the different reaction mechanisms. Initially, a description of multifragmentation was proposed as a process of successive binary splits [21] within the transitionstate model, or as an extension of the saddle-point interpretation to highly excited systems [22]. Later, several experimental data gave arguments for the large success of 
statistical models, and descriptions based on the phenomenology of phase transitions [23, 24, 25], so that binary splits might even appear among the possible fragment configurations. Not only light-ion induced reactions comply largely with the statistical nature of the process (ISIS experiments [18]), but also recent heavy-ion experiments at Fermi energy (INDRA [26, 27, 28, 29, 30, 31], MULTICS [32]) gave special prominence to such a picture. As a function of the violence of the collision, the bimodal distribution of the largest-fragment size is interpreted as a signal of the finite-size counterpart of the liquid-gas phase transition in nuclear matter [33, 34], which is associated to a finite latent heat [35]: the energy (and density) of the system and the size of the largest fragments are order parameters which rule the evolution of the system from a configuration where a large cluster survives at low excitation energy to a configuration where the system disassembles in small fragments of comparable size at high-excitation [36, 37]. It should be pointed out that a dedicated study of the effect of the Coulomb field on the fragmentation process further enriched this picture and revived the interest on the phenomenology of binary fission in highly excited systems. In particular, also the effect of Coulomb frustration applies: this is a rather general phenomenon originally introduced in condensed-matter studies to describe a large variety of systems, ranging from magnets on specific lattices to liquid crystals, from spin glasses to protein folding. The common trait of these systems is the absence of a state where all interaction energies are minimised simultaneously. In the nuclear fragmentation process, Coulomb frustration describes the situation when the long-range repulsive Coulomb field and the short-range attractive nuclear interaction can not be minimised simultaneously, and results in one additional phase transition process, from the liquid phase to fission. This phenomenon of Liquid-Fission transition is not associated to any latent heat. It was observed and detailed within the Liquid Gas Model [38, 39] as well as in phenomenological nuclear statistical models [35, 40].

\subsection{Purpose of this work}

The experimental study detailed therein analyses the kinematic, production and isotopic observables of the two decay modes, fission and multifragmentation, tagged by the production of a specific nuclide in the intermediate-mass region (located below half the mass of the projectile, and ranging down to lithium isotopes). In the case of fission, such nuclide is the lightest binary partner, the other being close to the mass of the projectile. In the case of multifragmentation, such nuclide is one of the several fragments of similar size produced by the division of the source and has only a fraction of the mass of the projectile. In both cases, indirectly or more directly, the experimental signature we obtain by the kinematics of the intermediate-mass fragments gives a good information on the size of the heaviest product in the reaction. Especially in the case of multifragmentation, the characteristics of the heaviest fragment have a fundamental role in carrying robust signals of the finite-size counterpart of the nuclear liquid-gas phase transition. From such premises, the main aim of this experimental work is threefold: 
(i) Analysis. We focus on the selection problematics for high-resolution inclusive experiments, and we propose a new analysis procedure for correlating production and kinematic observables.

(ii) Experimental results. We provide several inclusive intermediate-mass fragment observables, as a function of the decay process: we quantify both isotopic cross sections and invariant cross sections, concerning the production and the kinematics, respectively.

(iii) Discussion. Concerning the exit channel: we analyse the boost imparted to the partners of binary splits, we extract the distribution of fragments along the mass number, and we analyse the strong staggering which characterises the nuclide production. Concerning the connection between the entrance channel and the exit channel: from the kinematic observable, we propose a new inclusive indicator for the impact parameter and we illustrate a possible interpretation for the evolution of the kinematic observable as a function of the size of the fragments for the different decay modes.

\section{Experimental overview}

The present study focuses on a situation where the contribution of two processes to the decay, fission and multifragmentation, makes experimental conditions rather challenging. On the other hand, these experimental conditions are greatly simplified by the choice of relativistic energies: in the case of relativistic nuclear reactions induced by hadrons, the fraction of the bombarding energy which remains in the system appears almost completely as thermal energy, reflected in a mostly isotropic kinematics. Under these conditions, an inclusive approach based on the use of a magnetic spectrometer is at present a very well adapted solution for connecting the production observables to the kinematic observables: in particular, the production observables consist in the full isotopic identification, extended to the complete range of fragment sizes; the kinematic observables are the distribution of longitudinal velocities, measured for single nuclides, from the magnetic rigidity, with very high precision and without any threshold. Nevertheless, we show in the following that some difficulties still remain when rather heavy fragments are studied, because the analysis procedure we adopted, based on the disentangling of the full velocity distribution in more components, becomes limited.

\subsection{Spallation at the multifragmentation threshold}

A first inclusive technique of disentangling reaction processes on the basis of longitudinal-velocity spectra was introduced for distinguishing the average characteristics of the fission fragments from those of the evaporation residues [41, 42, in inverse-kinematic experiments at the FRagment Separator [43, 44] at GSI in Darmstadt. Concerning spallation at the multifragmentation threshold, still with the same facility and experimental approach, the kinematic and production properties of intermediate- 


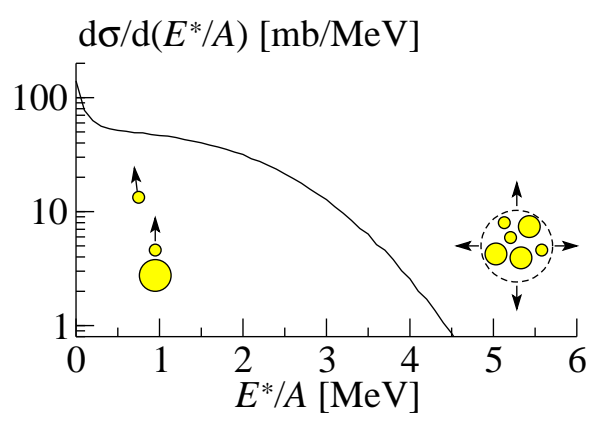

Figure 1. Intra-nuclear-cascade calculation of the distribution of excitation energy of the hot fragments in the system ${ }^{136} \mathrm{Xe}_{(1 \mathrm{AGeV})}+p$ (the intranuclear excitoncascade model of ref. [51] was used) Above around $3 \mathrm{MeV}$ per nucleon the onset of multifragmentation is expected. Elsewhere, fission-evaporation is the dominant decay process.

mass fragments were deduced in an inclusive experiment for the reaction ${ }^{56} \mathrm{Fe}+$ hydrogen at $1 \mathrm{GeV}$ per nucleon [45, 46]. With the purpose of reconstructing the invariantvelocity spectra of intermediate-mass fragments from inclusive kinematic observables, a simple procedure of analysis of the longitudinal-velocity spectra was introduced: it was based on the assumption that all exit channels were associated to the same value of the linear momentum transfer for one individual nuclide formed as a final product. The experimental results revealed mixed signals of multifragmentation and fission [45], which could however not be quantified in their relative share and identified separately; a possible disentangling of the two exit channels in terms of kinematic and thermal properties could only be suggested through the comparison with a statistical model. The same system was also the object of an exclusive experiment at ALADIN [47] at GSI. Profiting from particle correlations, but not reinforced by kinematic observables, it confirmed that intermediate-mass fragments were prevalently produced in binary decays, and also revealed the presence of a few decays with larger fragment multiplicity. Also in this case, the analysis of the reaction mechanism was discussed on the basis of statistical models.

In a more recent measurement at the FRagment Separator, the same inclusive experimental approach, previously used for iron, was followed to measure the system ${ }^{136} \mathrm{Xe}+$ hydrogen at $1 \mathrm{GeV}$ per nucleon [1]. ${ }^{136} \mathrm{Xe}$ is the stable nucleus with the largest neutron excess $N-Z$ among the nuclei below the Businaro-Gallone point [48, 49], where the saddle point becomes unstable to asymmetric splits. Binary decay should result in the production of neutron-rich intermediate-mass fragments. A large feeding of evaporation-fission decays should result from the range of excitation energies explored by this system. To corroborate our expectations, we calculate the excitation-energy distribution of the warm system excluding any cooling mechanism. As a rather general example for this kind of calculation, we employ the intranuclear exciton-cascade model of ref. [51]. In fig. 1, the excitation-energy distribution obtained for the warm system is shown, and it should be remarked that its range exceeds $3 \mathrm{MeV}$ per nucleon. According 
to the large amount of theoretical and experimental results [17], the transition towards multifragmentation is expected above this value and, similarly to fission, it should also manifest in the formation of neutron-rich intermediate-mass fragments. Since the reaction products from these two different channels feed the same region of the nuclear chart, isotopic cross sections alone are an insufficient information for studying the reaction mechanism. Two experimental strategies, based on coupling production and kinematics observables, are suited for this purpose. One is the exclusive strategy: its state of the art is the coupling of a $4 \pi$-detector with a spectrometer (at Fermi and lower energies: see ref. [50]) for probing both the initial excitation process and the excitation channel; in this case the isotopic identification is however limited to very small charges. The second strategy followed in the present work is the inclusive measurement of high-resolution velocity spectra of the entire distribution of fragments larger than alpha particles, identified in mass and charge; in this case a mayor limitation is the absence of event-by-event observables like correlations and the excitation.

\subsection{Inclusive event selection}

We detail in this paper the new method of inclusive event selection which we developed for the analysis of the system ${ }^{136} \mathrm{Xe}+p$. In comparison with the analysis method used for the system ${ }^{56} \mathrm{Fe}+p$, the new method has the advantage of being a quantitative technique of event selection, allowing to associate the probability for a given decay pattern to a group of events. In particular, the inclusive event selection method is used to identify kinematically the two processes of fission and multifragmentation in the formation of intermediate-mass fragments, and to quantify them in terms of nuclide cross sections and kinematic properties, benefiting from the two main specificities of the experimental method we employed. The first specificity is the possibility to measure small velocities in the projectile frame with high resolution, so as to access the physics of the small-energy portions of the kinetic-energy spectra of fragments; this portion is found in this work to give constraints for the identification of the exit channel. The second specificity is the possibility to connect such kinematic observable to the production of a given nuclide.

\subsection{The FRagment Separator}

The fragments were produced in inverse kinematics by directing a primary beam of ${ }^{136} \mathrm{Xe}$ at $1 \mathrm{~A} \mathrm{GeV}$ on a target of liquid hydrogen contained in a cryostat with thin titanium windows. The projectile residues were analysed in-flight, with the Fragment Separator [43, 44]. Four dipole magnets compose the magnetic spectrometer, a dispersive focal plane is localised behind the first two dipole magnets, and the full spectrometer was set achromatic. The identification of a fragment was deduced from the measurement of its time of flight, from recording the position where its trajectory intersects the dispersive focal plane, and from its nuclear charge measured in ionisation chambers, placed in the proximity of the terminal focal plane. Recoil velocities and kinetic energies were deduced from the magnetic rigidities, which were measured by the spectrometer with a relative 


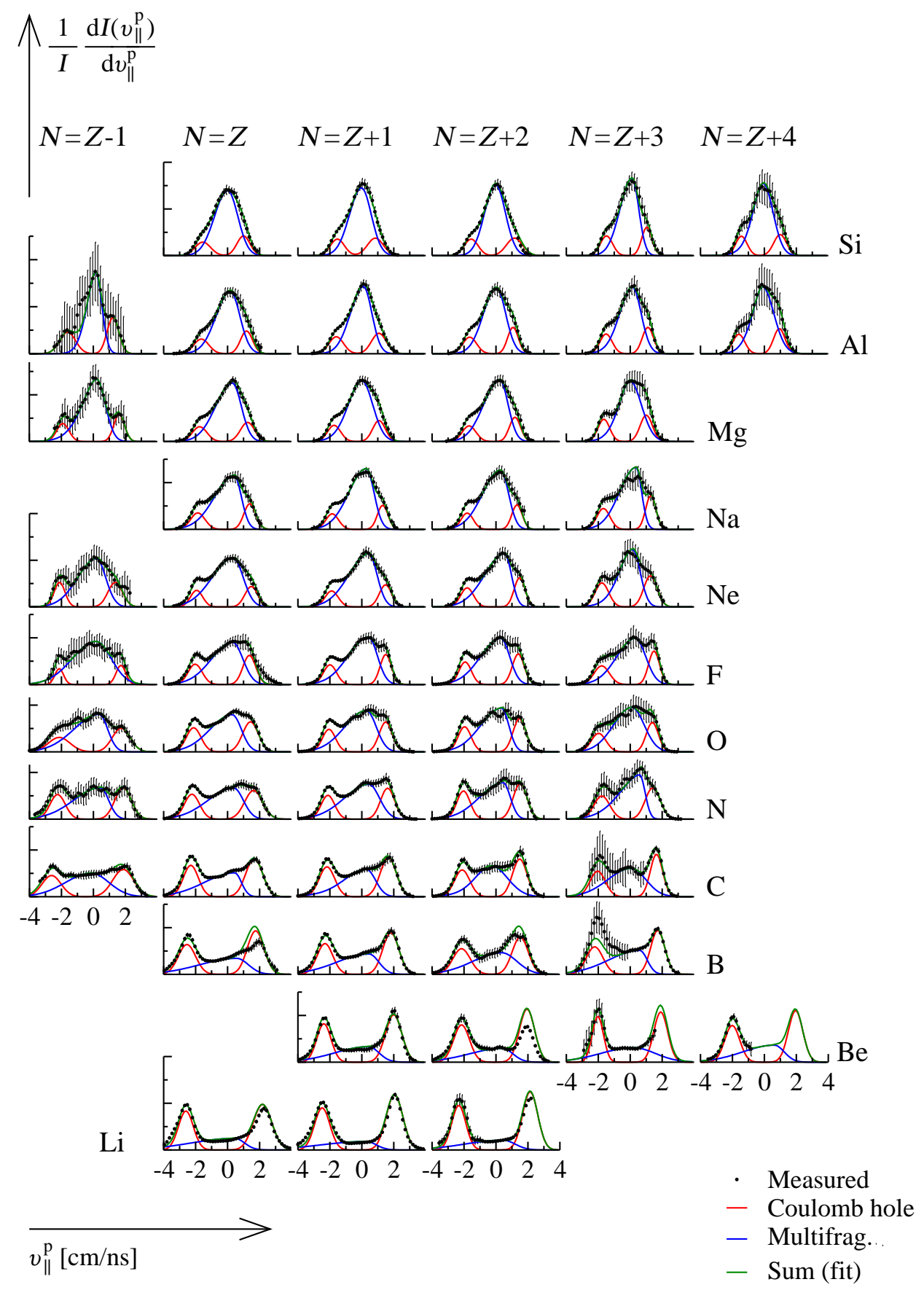

Figure 2. Systematics of measured velocity distributions as a function of the nuclide for elements ranging from $\mathrm{Li}$ to $\mathrm{Si}$, indicated with black dots; error bars show the statistical uncertainties. The measured spectra are disentangled by a fitting procedure (see section 3) in the contribution of a coulomb-hole mode and a multifragmentation mode (see section 44). The shapes of these two components, which are concave and convex, respectively, are deduced so that their sum is a fit of the measured shape.

uncertainty of $5 \cdot 10^{-4}$ (FWHM) for individual reaction products.

A series of corrections and calibrations were required for deducing the distribution 
of longitudinal recoil velocities for each nuclide. The raw spectrum was slightly corrected by taking into account the energy loss in the target. The events related to reactions in any layer of matter which does not correspond to hydrogen were suppressed; these events come for the greatest part from the material (mostly titanium) constituting the cryostat, and their contribution was measured with a specific experiment $₫$. The number of counts was normalised to the beam dose per target thickness, which was deduced by measuring the current of the primary beam. The data treatment required at this point to correct for the decrease of the angular transmission for ions with magnetic rigidities which deviate from the nominal values. This effect is seen in the velocity spectra, when they are constructed from composing different magnetic settings of the spectrometer: they present structures which appear periodically for each portion of the spectrum corresponding to one magnetic setting [45]. Since the angular transmission can be formulated simply as a function of the position of the trajectory intersecting the dispersive focal plane and the terminal focal plane [52], these structures are removed by correcting the data in a way that they correspond to the transmission of ions with nominal magnetic rigidities. Finally, additional corrections took into account the modification of the yields due to secondary reactions occurring in the hydrogen target and in other layers of matter intercepting the beam of fragments. These corrections are discussed in details in ref. [1] and they are the standard procedure of analysis for all nuclide-production measurements at the FRagment Separator.

At this stage, a distribution of measured events as a function of the longitudinal recoil velocity in the projectile frame $v_{\|}^{\mathrm{p}}$ is associated to each reaction product, identified in mass number and nuclear charge. The integral $I$ of each distribution is equal to the fraction of production cross section which is selected by the angular acceptance of the spectrometer. Fig. 2 shows a systematics of measured longitudinal-velocity spectra $\mathrm{d} I\left(v_{\|}^{\mathrm{p}}\right) / \mathrm{d} v_{\|}^{\mathrm{p}}$ normalised to the same integral for all measured isotopes of the elements ranging from lithium to silicon. In order to obtain invariant-cross-section distributions independently of the experimental conditions, the geometry of the angular acceptance of the spectrometer needs to be accurately accounted for in the data analysis.

In the following section, we describe the method for reconstructing the invariantcross-section distribution from the measured longitudinal-velocity spectra. Such technique is a further development of the method used in the analysis of the spallation of iron at $1 \mathrm{GeV}$ per nucleon measured at the FRagment Separator [45].

\section{Inclusive velocity spectra}

The FRagment Separator has a small angular acceptance of $\approx 15 \mathrm{mr}$ in the laboratory frame. This is of course a disadvantage (see discussions in refs. [1, 41, 42, 45, 52, 53]); on the other hand, even if the angular cuts should be accurately accounted for in the data

$\ddagger$ In particular, the present experimental technique imposed that several calibration experiments in identical conditions had to be repeated also with the empty titanium cryostat in order to exactly subtract any contribution from non-hydrogen materials [1] 
analysis, the combination of the reduced acceptance, the achromatic configuration and the relativistic beams has a special advantage as a counterpart: no magnetic aberration affects the resolution and all the focal planes (achromatic and dispersive) are fully defined. In particular, only two positions of a fragment trajectory (the intersections with the dispersive and the achromatic focal planes) are sufficient for achieving the nominal resolution of $5 \cdot 10^{-4}$ (FWHM) for the magnetic rigidity. For comparison, in a largeacceptance spectrometer, the counterpart of the broad angular acceptance is a complex form of the magnetic field, which demands to focus the analysis on an accurate trajectory reconstruction (as an example see refs. [54, 55]). The present experiment results in the reversed situation: in a small-acceptance achromat operated with relativistic beams, all the effort should be focused on the treatment of the angular cut by deconvoluting the momentum spectra of the fragments, but this procedure can be performed with high precision due to the well defined achromatic ion optics. Such precise knowledge of the ion optics is the key of our analysis procedure: it passes through the complex but conventional deconvolution of the inclusive velocity spectra which we detail below.

Before entering into the analysis, we also introduce some physical assumptions which will be used. The analysis procedure is based on the picture of the reaction process proceeding by a fast stage, characterised by nucleon-nucleon collisions, which ends up in an excited system, and a slower deexcitation phase [56]. The interaction with the target proton induces a mean shift and a broadening of the velocity distribution of the projectile. The shift is expected to be a function of the impact parameter [2]. The broadening is well represented by the Fermi momenta of the removed nucleons [57] and, within this picture, is isotropic [60]. In the course of the deexcitation, the system is considered thermalised. This justifies simplified assumptions on the angular distribution of the emitted particles. After thermalisation, the angular distributions of the particles emitted in sequential decay that reaches from evaporation of light particles up to symmetric fission, or produced by multifragmentation, are forwardbackward symmetric in the system of the emitting source. The angular anisotropy in the emission of light charged particles can be estimated within the Hauser-Feshbach picture [61], the anisotropy of fission processes can be estimated in the framework of the statistical population of the K-quantum numbers [62] and the anisotropic character of the multifragmentation process was already successfully described in early transport models [63, 64, 65]. However, since the angular momentum introduced in the collision phase is rather low and the excitation energy rather large, for the deconvolution procedure, we assume the angular distribution to be approximately isotropic for any decay process, in the frame of the decaying source. In agreement with this expectation, we add that the reaction analysed in this work is in between a large range of incident energies for which signatures of isotropy were measured in several experiments: from lowly-excited fissioning systems [13, 15] to spallation with high-energy light projectiles and relativistic heavy-ion collisions [19, 20, 66]). Still, we anticipate that we will find slight deviations from the isotropy in the data, which can not be described within our assumptions. It may not be helpless to also remind the following fundamental 
characteristic of any inclusive experiment: an isotropic emission pattern does not correspond necessarily to a symmetric distribution of longitudinal velocity. The latter is determined by an asymmetry in the distribution of the source energy or impact parameters, while the isotropic emission is a characteristic related to one single source sorted out of the whole distribution of the sources. This is also the principle of our kinematic analysis which consists of a conventional deconvolution procedure allowing to extract longitudinal-velocity distributions which are not necessarily symmetric by using an assumption of isotropic emission.

\subsection{Deconvolution procedure}

Once defined all this, we can go through the formalism, which is a conventional deconvolution procedure. It is sketched for the nuclide ${ }^{20} \mathrm{~F}$ in fig. 3 and is essentially based on the following three points, which resume the introductory discussion:

(i) Especially when dealing with relativistic protons, the kinematics of one individual nuclide can be decomposed in several isotropic emission patterns, each one centred around one apparent source $u$.

(ii) Each apparent source $u$ is localised at a longitudinal velocity $u^{\mathrm{p}}$ in the projectile frame (and $u^{\mathrm{L}}$ in the laboratory frame), which reflects the momentum transfer involved in the collision and is aligned along the beam axis: this assumption is equivalent to imposing that the overall kinematics is symmetric with respect to the beam axis.

(iii) The shape of the angular acceptance is known precisely.

The first two points are assumptions. The third point is an ingredient guaranteed by ion-optical calculations [52]. As described in section 2, these ion-optical calculations were also included in the procedure of raw-data treatment which had as an outcome the measured spectra of fig. 2, they are validated by requiring that the different magnetic settings of the spectrometer do not produce periodic structures on these spectra [45]; such a requirement is a tool to check and even to refine the exact properties of the angular acceptance.

We already defined the quantity $\mathrm{d} I\left(v_{\|}^{\mathrm{p}}\right) / \mathrm{d} v_{\|}^{\mathrm{p}}$ as the measured longitudinal-velocity spectrum in the projectile frame (see fig. 2); it is a sum of the contributions of sources complying to the hypotheses (1) and (2), and is affected by the angular-acceptance shape of the spectrometer. Additionally, for a given number of sources of velocity $u_{i}^{\mathrm{p}}$ in the projectile frame, since the velocities in the source frame are not relativistic, we define

$$
\sigma_{\mathrm{I} i}\left(v_{\|}^{\mathrm{p}}-u_{i}^{\mathrm{p}}\right)=\frac{\mathrm{d} \sigma}{\mathrm{d}\left(\boldsymbol{v}_{\|}^{\mathrm{p}}-\boldsymbol{u}_{i}^{\mathrm{p}}\right)}
$$

as the invariant cross section attributed to the source $i$. This is a production quantity which does not depend on the angular acceptance and, according to the assumption (1), 


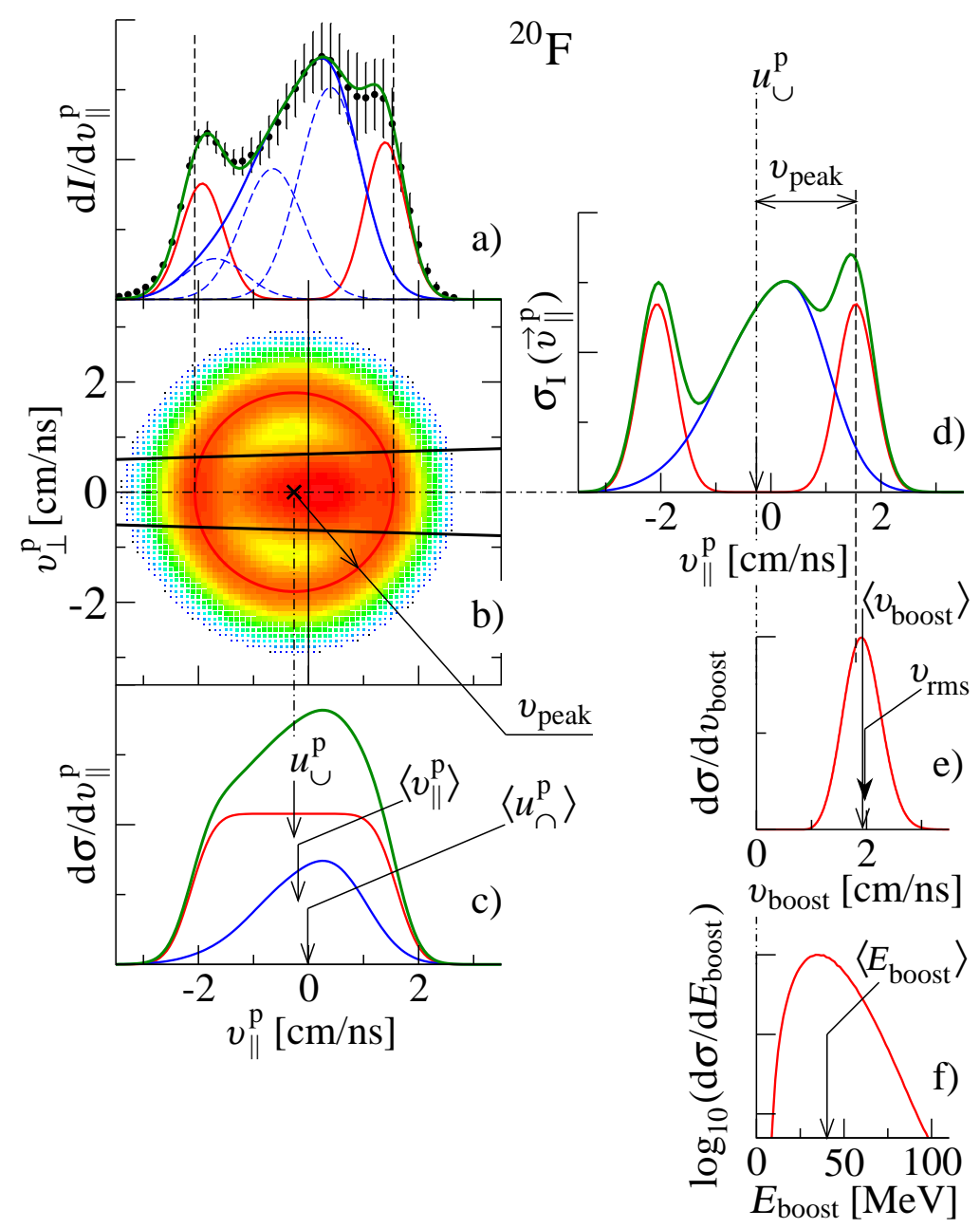

Figure 3. Procedure of reconstruction of the invariant cross sections applied to the nuclide ${ }^{20} \mathrm{~F}$. The decomposition in the concave component of the Coulomb-hole mode and the convex component of the multifragmentation mode indicated by different line types as in fig. 2. (a) Measured velocity distributions (black dots) and fitted spectra as in fig. 2, The decomposition of the multifragmentation mode in three Gaussian components is shown. (b) Planar cut along the beam axis of the reconstructed full distribution $\mathrm{d} \sigma / \mathrm{d} \boldsymbol{v}^{\mathrm{p}}$ in the beam frame as a cluster plot. The two lines indicate the boundaries of the angular acceptance, inside of which the fragments could be measured. The ridge of the Coulomb-hole component is indicated by the circle of radius $v_{\text {peak }}$. (c) Projection on the beam axis of the reconstructed full distribution $\mathrm{d} \sigma / \mathrm{d} \boldsymbol{v}^{\mathrm{p}}$. Arrows indicate the positions of the source of the Coulomb-hole component $u_{\sim}^{\mathrm{p}}$, the average source position of the multifragmentation component $\left\langle u_{\uparrow}^{\mathrm{p}}\right\rangle$ and the average longitudinal velocity $\left\langle v_{\|}^{\mathrm{p}}\right\rangle$. (d) Invariant-cross-section distribution. (e) Reconstructed cross-section distribution for the Coulomb-hole component as a function of the boost velocity in the source frame $v_{\text {boost }}$. The plot allows to deduce the mean boost $\left\langle v_{\text {boost }}\right\rangle$, which differs from $v_{\text {peak }}$ and from $v_{\text {rms }}$. (f) Reconstructed cross-section distribution for the Coulomb-hole component as a function of the total kinetic energy in the source frame $E_{\text {boost }} . v_{\mathrm{rms}}$ is deduced from the mean value $\left\langle E_{\mathrm{boost}}\right\rangle$.

it depends only on the absolute value of the relative velocity $\boldsymbol{v}_{\|}^{\mathrm{p}}-\boldsymbol{u}_{i}^{\mathrm{p}}$ in the frame of 
the source $u_{i}^{\mathrm{p}}$.

These cross sections are obtained by the deconvolution of the measured spectrum $\mathrm{d} I\left(v_{\|}^{\mathrm{p}}\right) / \mathrm{d} v_{\|}^{\mathrm{p}}$ taking into account the ion-optics required by the point (3). This procedure is detailed in a general form in appendix Appendix A. By composing these contributions, we construct the invariant-velocity cross section accounting for all sources, independently of the angular acceptance, which is denoted by

$$
\sigma_{\mathrm{I}}\left(\boldsymbol{v}^{\mathrm{p}}\right)=\frac{\mathrm{c}^{2}}{\mathrm{~m}^{2}} \frac{\mathrm{d} \sigma}{\mathrm{d} \boldsymbol{v}^{\mathrm{p}}}
$$

As explained in appendix Appendix A, we obtain the relation

$$
\sigma_{\mathrm{I}}\left(\boldsymbol{v}^{\mathrm{p}}\right)=\sum_{i} \sigma_{\mathrm{I} i}\left(\left|\boldsymbol{v}^{\mathrm{p}}-\boldsymbol{u}_{i}^{\mathrm{p}}\right|\right) .
$$

The spectrum reduces to a fully isotropic distribution if all emitting sources coincide for one individual nuclide.

This prescription was introduced in the simplified form of isotropic emission from one unique source in the analysis of the light residues in the system $\left.{ }^{56} \mathrm{Fe}_{(1 \mathrm{~A}} \mathrm{GeV}\right)+p$ [45]. For such system, no evidence was found for postulating a spreading of emitting sources. On the contrary, the description of multiple-source emission was necessary in the analysis of intermediate-mass fragments produced in the spallation of xenon.

\section{Results}

As shown in fig. 2, the evolution of the measured longitudinal-velocity spectra over a systematics of several light nuclides ranging from lithium to silicon invites to disentangle two main components: one has a shape with a concave centre boarded by two maxima: it corresponds to a Coulomb hole; the other component has a convex shape with a tail extending in the backward direction: as discussed in the following, we attribute to this mode the features of a multifragmentation process. The decomposition of the measured longitudinal-velocity distribution in the Coulomb-hole mode (symbol $\smile$ ) and the multifragmentation mode (symbol $\frown$ ) is shown in fig. 2 for elements ranging from lithium to silicon. The two kinematic modes are disentangleg by a fitting procedure which respects the constraints imposed by the detailed knowledge of the ion-optics. In the plot (a) of fig. 3 this decomposition is shown for ${ }^{20} \mathrm{~F}$. The convex shape of the multifragmentation mode is fitted as a skewed Gaussian distribution and the concave shape of the Coulomb-hole mode is fitted as the superposition of two Gaussian shapes with integrals constrained to have a ratio compatible with the effect of the angular acceptance on the forward and backward emission kinematics. The forward side of the spectra of the heavier isotopes of beryllium were not fully measured or partially affected because the magnetic rigidity of the primary beam was circumvented in the experiment; for these nuclides, the constraint on the concave shape of the Coulomb-hole mode allowed to profit from the precise measurement of the backward side, and the parameters for the convex shape of the multifragmentation mode were deduced from isobaric nuclides. 
The double-humped shape of the Coulomb-hole component, with integral $I_{\smile}\left(v_{\|}^{\mathrm{p}}\right)$, was interpreted as a shell in velocity space centred around one single isotropic emitting source of recoil velocity $u_{\smile}^{\mathrm{p}}$ in the beam frame. The plot (b) of fig. 3 shows a planar cut along the beam direction of the reconstructed velocity distribution $\mathrm{d} \sigma / \mathrm{d} \boldsymbol{v}^{\mathrm{p}}$; the radius $v_{\text {peak }}$ indicated in the figure is the radius of the ridge of the ring marking the Coulombhole component. The same figure shows that the distribution $\mathrm{d} \sigma / \mathrm{d} \boldsymbol{v}^{\mathrm{p}}$ is cut by the conical boundary of the acceptance so as to produce the two humps in the longitudinalvelocity spectra. The larger is the radius of the emission shell, the larger is the integral of the forward hump with respect to the backward hump. As the two peaks are spaced of a distance which largely exceeds their widths, their shapes are close to Gaussian distributions.

\subsection{Invariant cross sections}

We already anticipated that an isotropic emission pattern does not correspond necessarily to a symmetric distribution of longitudinal velocity. In other words, an asymmetric spectrum and an isotropic emission pattern are not two contradictory concepts. This is the case of the asymmetric shape of the multifragmentation component, of integral $I_{\curvearrowleft}\left(v_{\|}^{\mathrm{p}}\right)$. It could not be interpreted as the emission from one single isotropic source: we decomposed it in a set of Gaussian components $\mathrm{d} I_{i \curvearrowleft}\left(v_{\|}^{\mathrm{p}}\right) / \mathrm{d} v_{\|}^{\mathrm{p}}$ of different integrals, and different mean values $u_{i \sim}^{\mathrm{p}}$. We assumed that the emission kinematics is independent of the recoil velocity of the corresponding source $u_{i \sim}^{\mathrm{p}}$ : this assumption results in assigning to all the Gaussian components the same width, which is determined by the forward side of the convex shape of the multifragmentation mode (the effect of the angular acceptance on a Gaussian-like emission kinematics is discussed in ref. [52]). In the plot (a) of fig. 3, a decomposition of the multifragmentation mode in three Gaussian components is shown; for this spectrum, a number of three Gaussians was chosen so that their spacing is smaller than their width, sufficiently to avoid the appearing of structures.

By the fitting procedure, the measured spectrum is disentangled in the Coulombhole and multifragmentation components, so that

$$
\frac{\mathrm{d} I\left(v_{\|}^{\mathrm{p}}\right)}{\mathrm{d} v_{\|}^{\mathrm{p}}}=\frac{\mathrm{d} I_{\smile}\left(v_{\|}^{\mathrm{p}}\right)}{\mathrm{d} v_{\|}^{\mathrm{p}}}+\sum_{i} \frac{\mathrm{d} I_{i \curvearrowleft}\left(v_{\|}^{\mathrm{p}}\right)}{\mathrm{d} v_{\|}^{\mathrm{p}}} .
$$

All parameters involved in the fit have uncertainties deduced from the corresponding covariance matrix. The plot (b) of fig. 3 confirms that the backward-forward asymmetry which characterises the Coulomb-hole component in the measured spectra is produced by the angular acceptance of the spectrometer and disappears in the reconstructed distribution. On the contrary, the asymmetry of the multifragmentation component is not an effect of the acceptance and is kept in the reconstructed shape: this confirms that, as we anticipated, it depends on the reaction process.

The projection of the reconstructed distribution $\mathrm{d} \sigma / \mathrm{d} \boldsymbol{v}^{\mathrm{p}}$ on the beam axis is illustrated in the plot (c) of fig. 3. it corresponds to the longitudinal-velocity distribution 


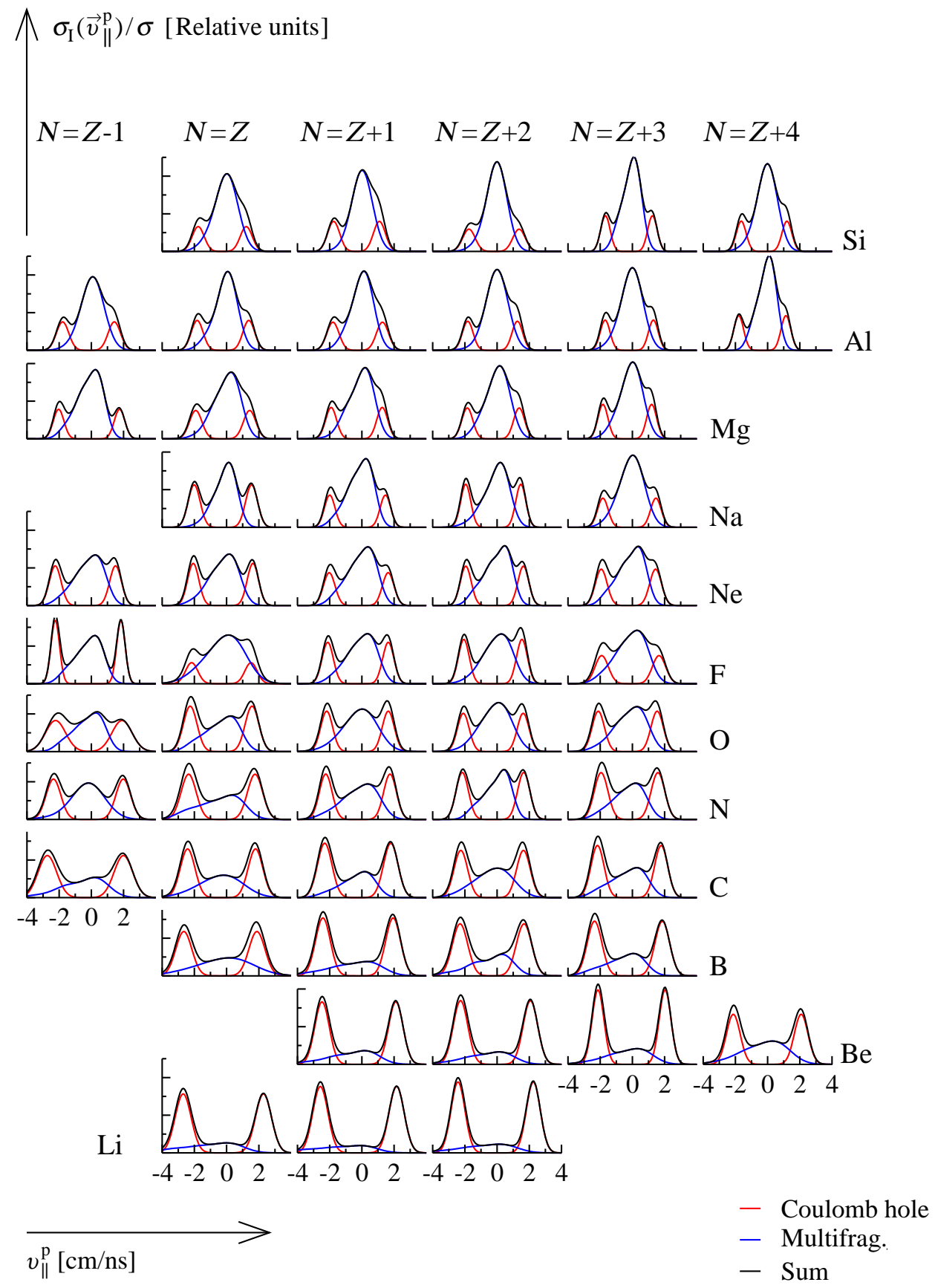

Figure 4. Systematics of invariant-cross-section distributions as a function of the nuclide for elements ranging from $\mathrm{Li}$ to Si decomposed in the Coulomb-hole and multifragmentation components. 
we would measure if the spectrometer had a full acceptance. In this representation the integrals of the different kinematic components give the corresponding production cross sections and their mean values give the average recoil of the corresponding sources.

The variation of the reconstructed quantity $\mathrm{d} \sigma / \mathrm{d} \boldsymbol{v}^{\mathrm{p}}$ along the beam axis coincides with the distribution of the invariant cross section $\sigma_{\mathrm{I}}\left(\boldsymbol{v}_{\|}^{\mathrm{p}}\right)$ illustrated in the plot (d) of fig. 3. The Coulomb-hole component $\sigma_{I_{\smile}}$ and the multifragmentation components $\sigma_{\mathrm{I}_{i}}$ of the distribution of invariant cross section are deduced from the corresponding components of the measured longitudinal-velocity spectra. The procedure of deconvolution requires the knowledge of the mean velocities of the sources $u_{\smile}^{\mathrm{p}}$ and $u_{i \curvearrowright}^{\mathrm{p}}$. These quantities are however not measured directly, but deduced through an optimisation procedure. The complete spectrum of invariant cross section is reconstructed by summing its components, shifted of the corresponding positions of the sources:

$$
\sigma_{\mathrm{I}}\left(\boldsymbol{v}_{\|}^{\mathrm{p}}\right)=\sigma_{\mathrm{I}}\left(v_{\|}^{\mathrm{p}}-u_{\smile}^{\mathrm{p}}\right)+\sum_{i} \sigma_{\mathrm{I}_{i}}\left(v_{\|}^{\mathrm{p}}-u_{\curvearrowleft}^{\mathrm{p}}\right),
$$

This is the application of Eq. (2) when $\boldsymbol{v}^{\mathrm{p}}$ is constrained to vary along the beam axis. The procedure of velocity reconstruction was applied for all intermediate-mass fragments ranging from lithium to silicon. In fig. 4 the resulting systematics of invariantcross-section distributions is shown, deduced from the deconvolution of the measured longitudinal-velocity spectra illustrated in fig. 2, The main parameters characterising the reconstructed spectra are listed in table B1 in the appendix Appendix B.

One more representation of the Coulomb-hole component is obtained by integrating the corresponding reconstructed distribution $\mathrm{d} \sigma / \mathrm{d} \boldsymbol{v}^{\mathrm{p}}$ over the polar angle. This is shown in the plot (e) of fig. 3. it probes the evolution of the cross section as a function of the Coulomb boost $v_{\text {boost. }}$. The plot (f) of fig. 3 is an equivalent representation in terms of the total kinetic energy $E_{\text {boost }}$. The mean value $\left\langle E_{\text {boost }}\right\rangle$ is indicated in the plot (f) and corresponds to the velocity $v_{\text {rms }}$ indicated in the plot (e). $v_{\text {rms }}=\sqrt{\left\langle v_{\text {boost }}\right\rangle^{2}+V}$ accounts for the variance $V$ of the velocity distribution and is slightly larger than the mean velocity $\left\langle v_{\text {boost }}\right\rangle$, also indicated in the plot (e). In section 5.1 the quantity $v_{\text {rms }}$ is compared to fission velocities calculated under different prescriptions for the Coulomb boost.

\subsection{Production cross sections}

The production cross sections were deduced from integrating the reconstructed distributions $\mathrm{d} \sigma / \mathrm{d} \boldsymbol{v}^{\mathrm{p}}$. The disentangling of the velocity spectra into the two kinematic modes allowed to separate the contributions of each mode. The results are presented for the isotopic chains of elements ranging from lithium to silicon in fig. 5 and listed in table 1. The uncertainties account for statistical and systematical contributions (see ref. [1] for details). The production of the lightest elements is mostly related to the Coulomb-hole mode. For heavier elements the multifragmentation mode gains gradually a larger fraction of cross section and it equals the Coulomb-hole mode around silicon, 
where the method of deconvolution reaches its limit of applicability. This evolution can be appreciated in the element and neutron-number distributions of cross sections in fig. 6. The average neutron-to-proton ratio $\langle N / Z\rangle$ of the intermediate-mass fragments produced by the two modes are indistinguishable, as shown in fig. 7? both the two modes feed prevalently the neutron-rich side of the nuclide chart with very similar shapes of the cross-section distributions.

\subsection{Limitations of the method}

It was not possible to extend the deconvolution for heavier elements than silicon because the uncertainties of the fitting parameters for the identification of the two kinematic modes in the measured velocity spectra determined a limit for the analysis. Above silicon, a simplified method was employed, based on the assumption of one unique isotropic source associated to the kinematics of one fragment [1]; the same simplification was also employed in the analysis of the kinematics of the intermediate-mass fragments formed in the system ${ }^{56} \mathrm{Fe}_{(1 A \mathrm{GeV})}+p$ [45].

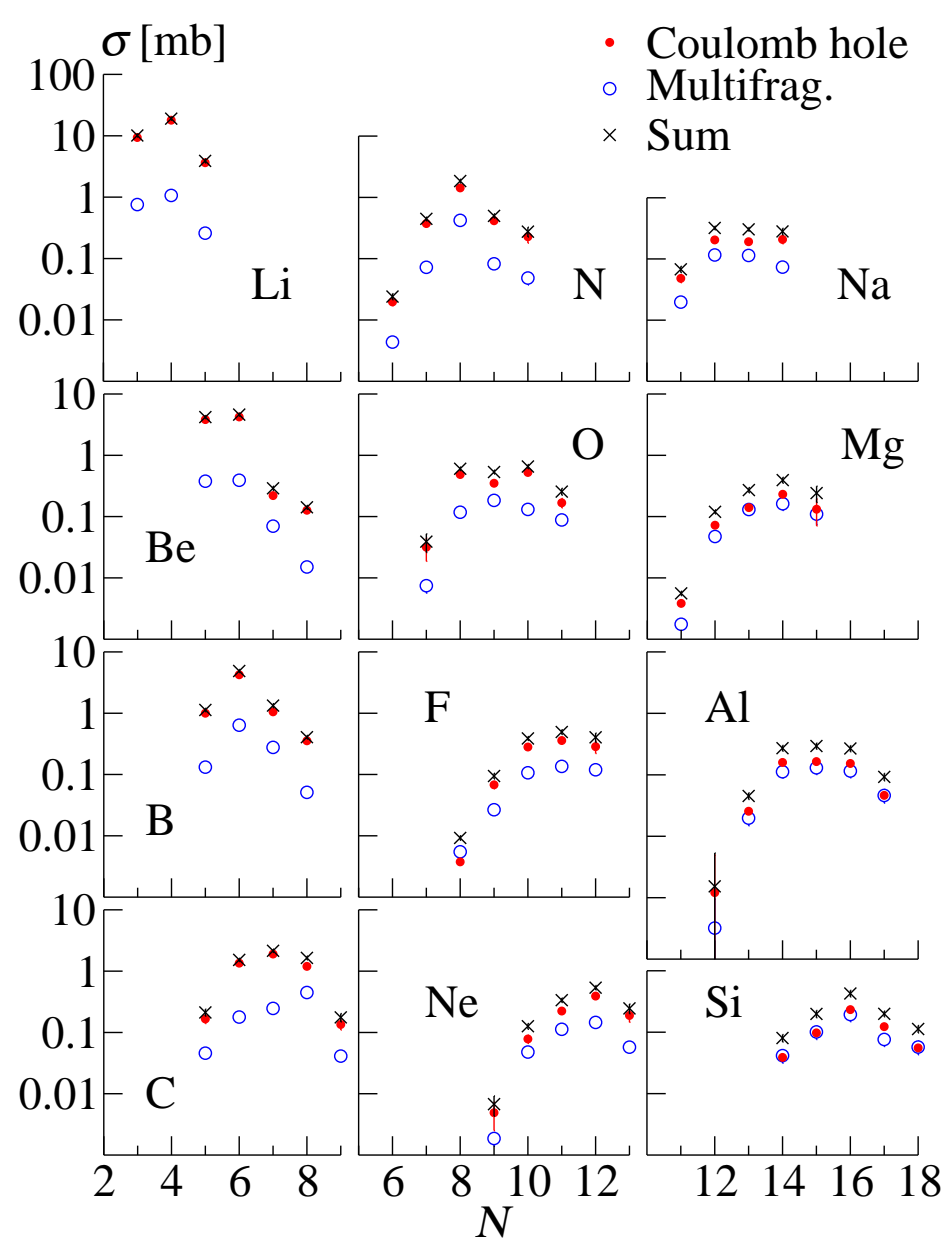

Figure 5. Production cross sections for the isotopes of elements ranging from Li to Si. The contributions from the two kinematic modes are shown. 


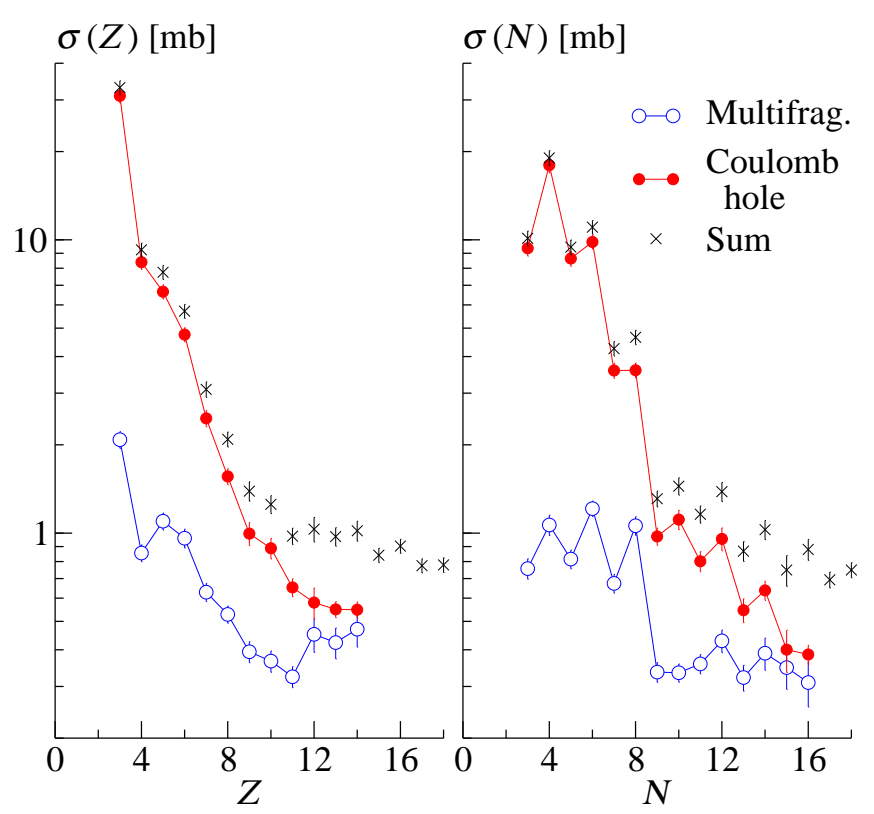

Figure 6. Element (left) and neutron-number (right) distributions of the intermediate-mass-fragment cross sections. The contribution from the two kinematic modes are indicated.

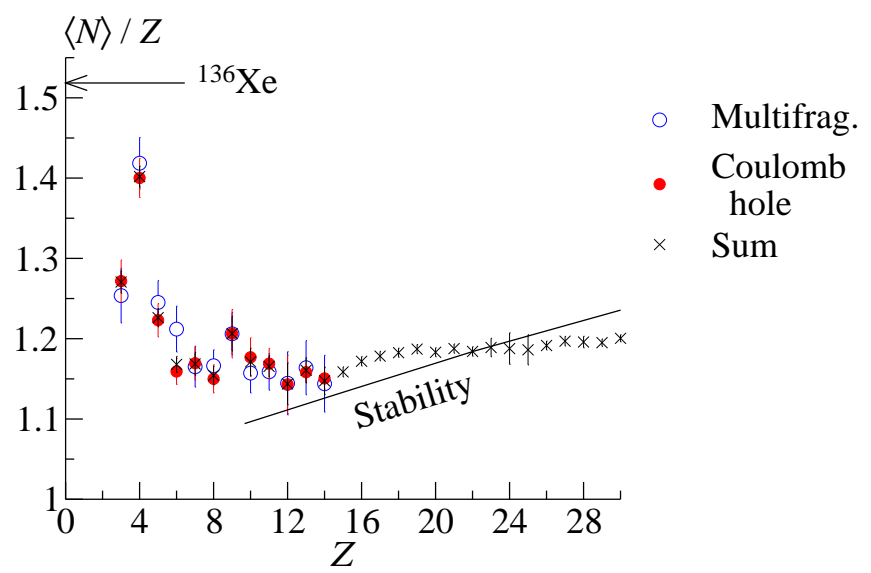

Figure 7. Evolution of the average neutron-to-proton ratio $\langle N / Z\rangle$ of the intermediate-mass fragments as a function of the element number. The contributions from the two kinematic modes are indicated.

Concerning the multifragmentation mode, we already pointed out the presence of a tail extending to the backward direction which characterises the lightest fragments. Such tail should result from the early stages of the collision and reflects fluctuations in the momentum transfer: following an intranuclear-cascade picture, these fluctuations are connected to a smaller or larger interaction of the shower of ejectile nucleons with the system, and result in a distribution of emitting sources. This distribution would be attributed to the longitudinal momentum transfer due to friction, while the shape of the isotropic distribution would be governed by the Fermi momenta of the emitted 
Table 1. Nuclide cross sections $\sigma$ measured in this work for the production of elements ranging from $\mathrm{Li}$ to $\mathrm{Si}$. The cross section restricted to the Coulomb-hole or multifragmentation mode is indicated as $\sigma_{\smile}$ or $\sigma_{\curvearrowleft}$, respectively.

\begin{tabular}{|c|c|c|c|c|c|c|c|}
\hline$A$ & $\sigma[\mathrm{mb}]$ & $\sigma_{\smile}[\mathrm{mb}]$ & $\sigma_{\curvearrowleft}[\mathrm{mb}]$ & $A$ & $\sigma[\mathrm{mb}]$ & $\sigma_{\smile}[\mathrm{mb}]$ & $\sigma_{\curvearrowleft}[\mathrm{mb}]$ \\
\hline \multicolumn{8}{|c|}{10 (200/(2) } \\
\hline 6 & $10.12(59)$ & $9.36(56)$ & $0.757(60)$ & & & $0.281(23)$ & $0.1066(93)$ \\
\hline 7 & $19.0 \pm 1.1$ & $18.0 \pm 1.1$ & $1.066(85)$ & 20 & $0.494(44)$ & $0.358(39)$ & $0.136(13)$ \\
\hline 8 & $3.90(30)$ & $3.64(29)$ & $0.259(33)$ & 21 & $0.404(70)$ & $0.285(65)$ & $0.119(22)$ \\
\hline & $\mathrm{Be}$ & & & \multicolumn{4}{|c|}{$\mathrm{Ne}$} \\
\hline 9 & $4.20(25)$ & $3.82(23)$ & $0.378(32)$ & 19 & $0.0068(24)$ & $0.0049(24)$ & $0.00186(47)$ \\
\hline 10 & $4.61(28)$ & $4.22(26)$ & $0.392(32)$ & 20 & $0.126(13)$ & $0.078(12)$ & $0.0479(46)$ \\
\hline 11 & $0.290(23)$ & $0.220(19)$ & $0.070(10)$ & 21 & $0.336(28)$ & $0.223(21)$ & $0.113(14)$ \\
\hline 12 & $0.142(10)$ & $0.1267(94)$ & $0.0151(20)$ & 22 & $0.538(44)$ & $0.392(36)$ & $0.146(19)$ \\
\hline & B & & & 23 & $0.247(45)$ & $0.189(43)$ & $0.058(11)$ \\
\hline 10 & $1.131(73)$ & $0.999(66)$ & $0.132(18)$ & \multicolumn{4}{|c|}{$\mathrm{Na}$} \\
\hline 11 & $4.88(29)$ & $4.25(26)$ & $0.638(46)$ & 22 & $0.0677(80)$ & $0.0480(71)$ & $0.0197(28)$ \\
\hline 12 & $1.33(10)$ & $1.055(89)$ & $0.277(32)$ & 23 & $0.321(22)$ & $0.205(13)$ & $0.116(14)$ \\
\hline 13 & $0.406(27)$ & $0.355(25)$ & $0.0512(55)$ & 24 & $0.305(21)$ & $0.191(12)$ & $0.114(14)$ \\
\hline & $\mathrm{C}$ & & & 25 & $0.282(35)$ & $0.208(32)$ & $0.074(10)$ \\
\hline 11 & $0.212(28)$ & $0.166(26)$ & $0.0459(75)$ & \multicolumn{4}{|c|}{$\mathrm{Mg}$} \\
\hline 12 & $1.528(92)$ & $1.350(84)$ & $0.178(13)$ & 23 & $0.00559(46)$ & $0.00384(29)$ & $0.00175(29)$ \\
\hline 13 & $2.15(13)$ & $1.90(12)$ & $0.247(27)$ & 24 & $0.1196(91)$ & $0.0723(46)$ & $0.0473(65)$ \\
\hline 14 & $1.65(12)$ & $1.197(89)$ & $0.449(52)$ & 25 & $0.271(27)$ & $0.1400(87)$ & $0.131(23)$ \\
\hline 15 & $0.176(27)$ & $0.135(25)$ & $0.0412(80)$ & 26 & $0.394(44)$ & $0.232(20)$ & $0.162(36)$ \\
\hline & $\mathrm{N}$ & & & 27 & $0.242(72)$ & $0.132(62)$ & $0.110(36)$ \\
\hline 13 & $0.0241(28)$ & $0.0197(27)$ & $0.00436(59)$ & \multicolumn{4}{|c|}{$\mathrm{Al}$} \\
\hline 14 & $0.446(33)$ & $0.374(30)$ & $0.0723(62)$ & 25 & $0.0015(38)$ & $0.0012(38)$ & $0.00032(63)$ \\
\hline 15 & $1.85(13)$ & $1.42(11)$ & $0.422(36)$ & 26 & $0.0453(56)$ & $0.0255(22)$ & $0.0197(49)$ \\
\hline 16 & $0.499(38)$ & $0.416(35)$ & $0.0823(87)$ & 27 & $0.271(29)$ & $0.159(14)$ & $0.112(24)$ \\
\hline & $0.276(50)$ & $0.228(48)$ & $0.0020(01)$ & 28 & $0.294(34)$ & $0.164(14)$ & $0.130(29)$ \\
\hline & $\mathrm{O}$ & & & 29 & $0.268(31)$ & $0.153(14)$ & $0.115(26)$ \\
\hline 15 & $0.039(13)$ & $0.032(13)$ & $0.0075(18)$ & 30 & $0.092(13)$ & $0.0463(38)$ & $0.046(12)$ \\
\hline 16 & $0.603(40)$ & $0.485(35)$ & $0.118(10)$ & \multicolumn{4}{|c|}{$\mathrm{Si}$} \\
\hline 17 & $0.534(38)$ & $0.350(28)$ & $0.184(19)$ & \multicolumn{2}{|c|}{$28 \quad 0.080(11)$} & $0.0388(33)$ & $0.042(10)$ \\
\hline 18 & $0.656(50)$ & $0.525(45)$ & $0.131(12)$ & 29 & 0.199(27) & $0.0977(81)$ & $0.101(25)$ \\
\hline & $0.256(33)$ & $0.168(30)$ & $0.088(11)$ & \multirow{2}{*}{$\begin{array}{l}30 \\
31\end{array}$} & \multirow{2}{*}{$\begin{array}{l}0.427(53) \\
0.199(22)\end{array}$} & \multirow{2}{*}{$\begin{array}{l}0.233(21) \\
0.123(10)\end{array}$} & \multirow{2}{*}{$\begin{array}{l}0.194(46) \\
0.076(18)\end{array}$} \\
\hline & $\mathrm{F}$ & & & & & & \\
\hline 17 & $0.00925(79)$ & $0.00376(23)$ & $0.00549(69)$ & \multirow[t]{2}{*}{32} & \multirow[t]{2}{*}{$0.113(16)$} & \multirow[t]{2}{*}{$0.0555(47)$} & \multirow[t]{2}{*}{$0.057(14)$} \\
\hline 18 & $0.095(10)$ & $0.0678(91)$ & $0.0266(28)$ & & & & \\
\hline
\end{tabular}


nucleons [57] and the recoil in the decay process (fission or multifragmentation). It must be admitted, however, that the relatively small angular acceptance does not allow to get direct experimental information on the shape of the velocity distribution in transverse direction. Guided by the present knowledge from other experiments and in agreement with the theoretical expectations for the contributions from Fermi momenta and decay recoil, we still keep the underlying assumption of isotropic emission patterns as a technical option to describe the tail to backward velocities. However, the validity of this assumption may only be tested by a full-acceptance experiment.

\section{Discussion}

In the analysis of the velocity spectra of the intermediate-mass fragments formed in the system ${ }^{56} \mathrm{Fe}_{(1 A \mathrm{GeV})}+p$ [45] similar results were found: the effect of the Coulomb field on the kinematics of fragments reveals the interplay of a Coulomb-hole mode and a multifragmentation mode, as shown in the left column of fig. 8. However, in such system the Coulomb-hole mode exhibits two humps with large widths, with the consequence that the two modes could not be disentangled in single spectra and they were identified only for the extreme case when the contribution of one component is dominant; their coexistence in the production of one individual nuclide could only be postulated on the basis of a model calculation, as shown in the right column of fig. 8. The calculation, where the distribution of hot fragments is evaluated with the intranuclear exciton-cascade model [51], and their breakup is simulated by the Statistical Multifragmentation Model [58, 59, 23], associates the Coulomb-hole component in the velocity spectrum to events with multiplicity of larger fragments than alpha $M=2$ and the multifragmentation component to events with multiplicity $M>2$.

On the contrary, for the heavier system ${ }^{136} \mathrm{Xe}_{(1 A \mathrm{GeV})}+p$, the identification of the two distinct channels in the formation of intermediate-mass fragments is an experimental result, even when they coexist in the formation of the same nuclide. To our knowledge, there have not been previous attempts to extract this observable from inclusive experiments and for similar systems.

\subsection{Exit channel}

5.1.1. Coulomb hole. Double-humped spectra were measured at the FRagment Separator in several experiments: they were usually taken as the signal of binary fission characterised by mostly symmetric splits (without being exhaustive, we cite refs. [41, 42]), and were also observed in presence of asymmetric fission splits, when light fragments were measured [53]. With the purpose of comparing to fission data it is therefore interesting to quantify the Coulomb repulsion reflected by the forward and backward peaks in the present data. In fig. 9, the boost $v_{\text {rms }}$ is deduced as in the plot (e) of fig. 3 and its evolution is studied as a function of the neutron number for elements ranging from lithium to silicon. The same experimental points are collected in table 2 . 
As described in section 4.1, $v_{\text {rms }}$ is the velocity deduced from the mean value of the total kinetic energy distribution $\left\langle E_{\text {boost }}\right\rangle$, which represents the average mechanism leading to the observed nucleus. Because of the wide velocity spectra, this quantity results slightly larger than the mean value of the velocity distribution $\left\langle v_{\text {boost }}\right\rangle$, which is also plotted in fig. 9 for comparison. In the figure, the reconstructed boost $v_{\text {rms }}$ is compared to the fission velocity calculated under different prescriptions, all applied to the same extreme case: the binary split of the largest possible mother nucleus ${ }^{136} \mathrm{Xe}$, which also determines the largest boost. The calculations do not change sensibly for the split of lighter isotopes of xenon, if fission occurred after the emission of some neutrons. Three prescriptions are tested. First, we compare to the empirical systematics of total kinetic energy for light fissioning nuclei condensed in the parametrisation of Beck and Szanto de Toledo [67, and further adapted to describe asymmetric splits by using the prescription of Ref. [45]. Then, we compare to the scission-point model of Wilkins et al., which was already used to describe similar data [53]. The model deduces the total kinetic energy from an empirical liquid-drop description, based on the parametrisation of Refs. [68, 69]. The underlying picture of the model, which is the fission of a deformed system through the formation of a neck, is appropriate to describe the symmetric split of heavy fissioning systems, but is not sufficiently justified when the formation of intermediate-mass fragments is described. This second calculation may be regarded as a lower limit. As a third option, we deduce the boost from the nuclear potential which the light fragment and its heavy partner would explore in a fusion reaction, according to the empirical model of Bass [70, 71]:

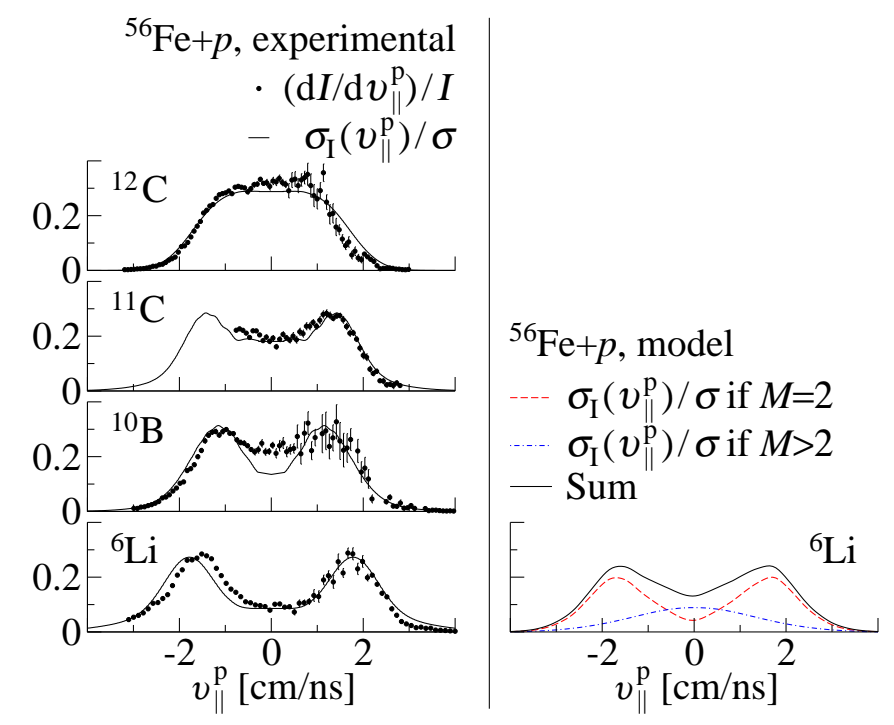

Figure 8. Left column. Measured velocity distributions and invariant-cross-section distributions for four nuclides produced in ${ }^{56} \mathrm{Fe}_{(1 A \mathrm{GeV})}+p$. Right column. Invariantcross-section distribution for ${ }^{6} \mathrm{Li}$, calculated with the intranuclear exciton-cascade model [51, coupled with the Statistical Multifragmentation Model [58, 59, 23]; two components, associated to the multiplicity $M=2$ and $M>2$ (for larger fragments than alpha) are indicated. Data and calculation from [45]. 
within this more appropriate description, the Coulomb repulsion acts on non-deformed fission fragments and results in the largest boost, compared to the first two prescriptions. For the extreme case of a fissioning ${ }^{136} \mathrm{Xe}$, while isotopes from nitrogen to neon are well described both by the fission systematics and the Bass potential, the heavier isotopes from sodium to silicon could be compatible with a lighter fissioning system, according to the same two prescriptions, or still with the split of ${ }^{136} \mathrm{Xe}$ within the scission-point model of Wilkins et al. The large increase of the boost $v_{\text {rms }}$ for decreasing element numbers in the region from lithium to carbon, is not even described by the Bass potential. This phenomenology is rather general: the same conclusion was presented for the analysis of the system $\left.{ }^{56} \mathrm{Fe}_{(1 \mathrm{~A}} \mathrm{GeV}\right)+p$ [45] and a similar tendency was found for the fission fragments in the system ${ }^{238} \mathrm{U}_{(1 \mathrm{~A} G \mathrm{GV})}+p$ [53]. Even the simultaneous emission of a third

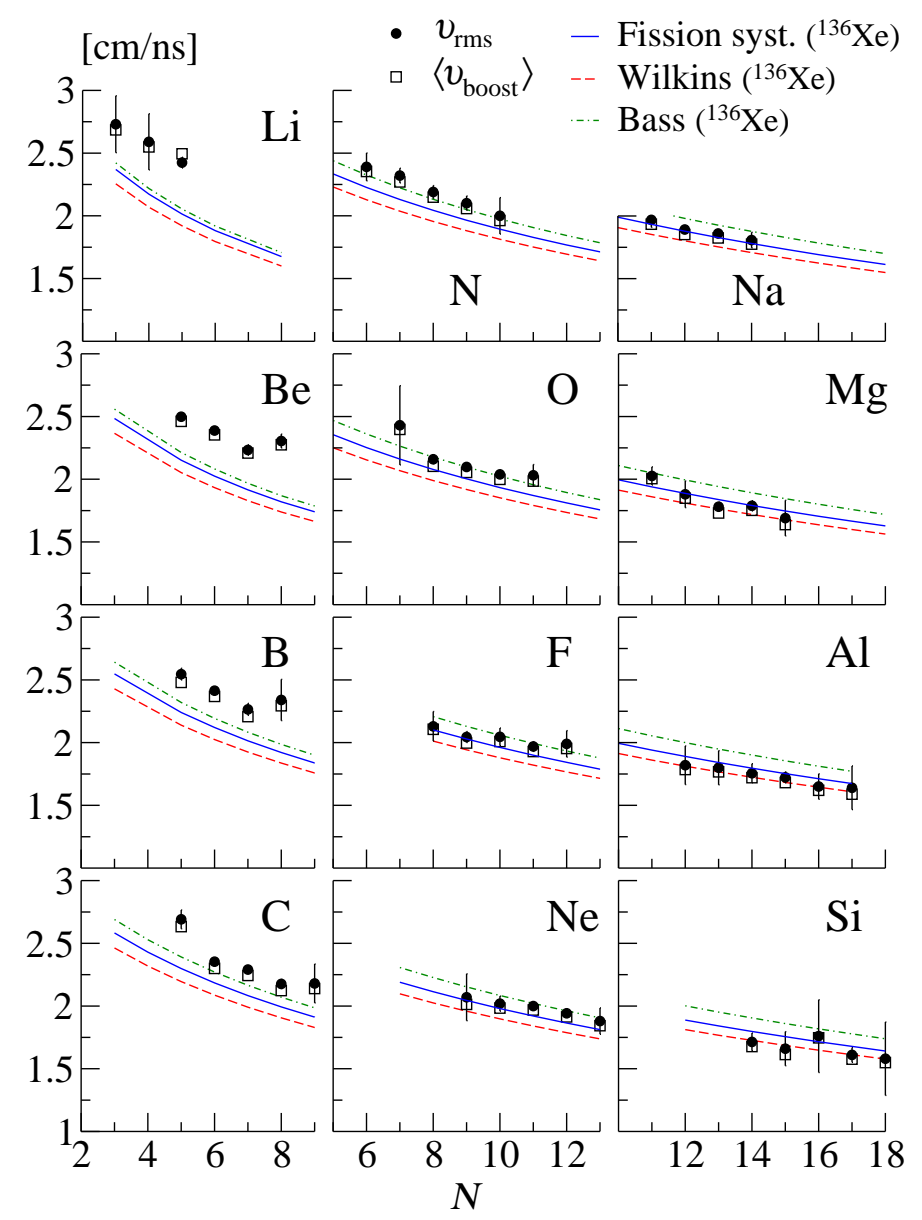

Figure 9. Evolution of the reconstructed boost $v_{\text {rms }}$ of the Coulomb-hole mode (and of the mean boost $\left\langle v_{\text {boost }}\right\rangle$, plotted for comparison, without error bars) as a function of the neutron number, for elements ranging from lithium to silicon. Three prescriptions for calculating the Coulomb boost in the binary split of ${ }^{136} \mathrm{Xe}$ are compared to the measurement: the total-kinetic-energy systematics of Beck and Szanto de Toledo 67, the scission-point model of Wilkins et al. [68, 69] and the nucleus-nucleus fusion model of Bass [70, 71]. 
Table 2. Reconstructed boost $v_{\mathrm{rms}}$, deduced for the Coulomb-hole mode. The uncertainty, indicated in parenthesis, includes both statistical and systematical errors.

\begin{tabular}{|c|c|c|c|c|c|c|c|}
\hline$A$ & $v_{\mathrm{rms}}$ & $A$ & $v_{\mathrm{rms}}$ & $A$ & $v_{\mathrm{rms}}$ & $A$ & $v_{\mathrm{rms}}$ \\
\hline & $\mathrm{Li}$ & 13 & $2.291(31)$ & 18 & $2.045(44)$ & 24 & $1.88(11)$ \\
\hline 6 & $2.73(23)$ & 14 & $2.176(37)$ & 19 & $2.046(70)$ & 25 & $1.781(37)$ \\
\hline 7 & $2.59(22)$ & 15 & $2.18(16)$ & 20 & $1.969(39)$ & 26 & $1.788(40)$ \\
\hline 8 & $2.424(43)$ & & $\mathrm{N}$ & 21 & $1.99(11)$ & 27 & $1.69(14)$ \\
\hline & $\mathrm{Be}$ & 13 & $2.39(11)$ & & $\mathrm{Ne}$ & & $\mathrm{Al}$ \\
\hline 9 & $2.498(15)$ & 14 & $2.321(58)$ & 19 & $2.07(19)$ & 25 & $1.82(15)$ \\
\hline 10 & $2.388(20)$ & 15 & $2.190(51)$ & 20 & $2.019(60)$ & 26 & $1.80(14)$ \\
\hline 11 & $2.233(42)$ & 16 & $2.101(57)$ & 21 & $2.000(32)$ & 27 & $1.756(76)$ \\
\hline 12 & $2.305(55)$ & 17 & $2.00(15)$ & 22 & $1.942(32)$ & 28 & $1.723(45)$ \\
\hline & B & & $\mathrm{O}$ & 23 & $1.88(11)$ & 29 & $1.65(10)$ \\
\hline 10 & $2.546(47)$ & 15 & $2.43(32)$ & & $\mathrm{Na}$ & 30 & $1.64(17)$ \\
\hline 11 & $2.414(20)$ & 16 & $2.159(35)$ & 22 & $1.969(59)$ & & $\mathrm{Si}$ \\
\hline 12 & $2.265(48)$ & 17 & $2.098(21)$ & 23 & $1.892(16)$ & 28 & $1.714(71)$ \\
\hline 13 & $2.34(16)$ & 18 & $2.039(33)$ & 24 & $1.860(20)$ & 29 & $1.66(14)$ \\
\hline & $\mathrm{C}$ & 19 & $2.031(86)$ & 25 & $1.808(62)$ & 30 & $1.76(29)$ \\
\hline 11 & $2.691(75)$ & & $\mathrm{F}$ & & $\mathrm{Mg}$ & 31 & $1.610(59)$ \\
\hline 12 & $2.354(23)$ & 17 & $2.13(12)$ & 23 & $2.026(73)$ & 32 & $1.58(29)$ \\
\hline
\end{tabular}

light fragment or particle would not deform the shape of the Coulomb-hole component sensibly. The large value of the Coulomb boost $v_{\text {rms }}$ may manifest the presence of some additional contribution to the total kinetic energy.

As a general conclusion of this section, we relate the Coulomb-hole mode to events with low fragment multiplicity and large mass asymmetry $\left(A_{1}-A_{2}\right)\left(A_{1}+A_{2}\right)$, where $A_{1}$ is the mass number of the heaviest fragment and $A_{2}$ indicates the second heaviest fragment, the one the velocity spectrum belongs to. The large width of the backward and forward peaks $\left\langle\sigma_{\| \text {peak }}^{\mathrm{p}}\right\rangle$, plotted in fig. 10 for $A \leq 30$, is compatible with a large number of decay paths all ending in the production of the observed nuclide; these decay paths account for several different breakup configurations and different quantities of particles which, depending on the excitation energy of the system, can be evaporated before and after the breakup.

5.1.2. Multifragmentation The multifragmentation mode extends over a large range of recoil velocities and indicates that the kinetic energy is shared among a large multiplicity of fragments with small mass asymmetry. In the analysis of the system ${ }^{56} \mathrm{Fe}_{(1 \mathrm{AGeV})}+p$ such a shape was proposed as a signature compatible with multifragmentation. In such system, which has a larger excitation energy per nucleon than ${ }^{136} \mathrm{Xe}+p$, the multifragmentation mode was found to predominate already above carbon [45]. Along 


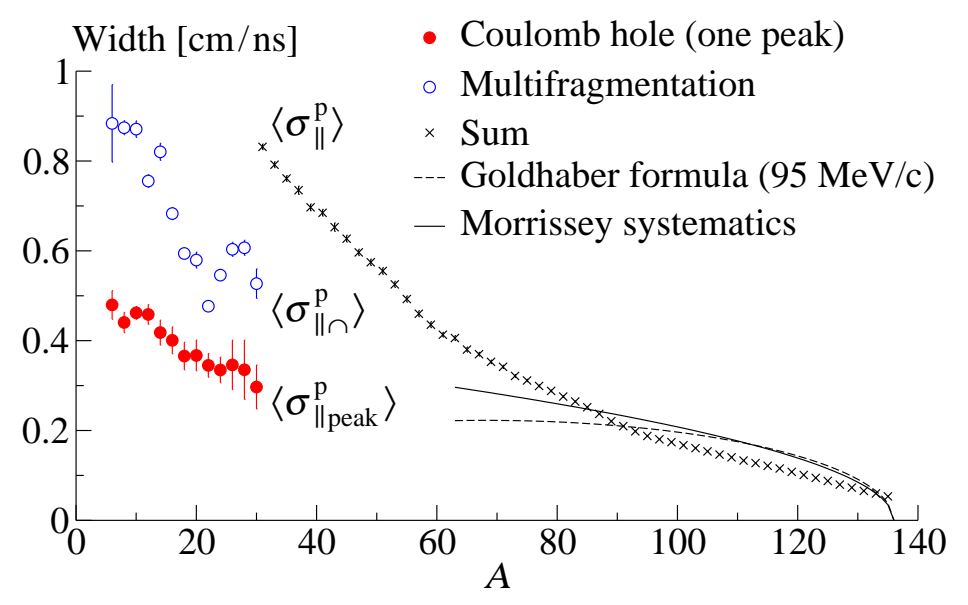

Figure 10. Evolution as a function of the mass number of the residue of the mean standard deviation of the invariant-cross-section distribution averaged on the atomic number. For lower masses than $A=30$ the width associated to the forward side of the multifragmentation component and the width of each peak of the Coulomb-hole mode are shown. The mean standard deviation of the full spectrum is shown for masses heavier than $A=30$. The systematics of Morrissey 60 and the prescription of Goldhaber [57] are shown for comparison.

the same line, in agreement with the picture that the multifragmentation share increases with the excitation of the system, inclusive measurements at the incident energy of 1 $\mathrm{GeV}$ per nucleon found only a small contribution from the Coulomb-hole component in comparison with the multifragmentation mode: it was the case of the systems ${ }^{56} \mathrm{Fe}+{ }^{\text {nat }} \mathrm{Ti}$ [45], ${ }^{136} \mathrm{Xe}+{ }^{\text {nat }} \mathrm{Ti}[72],{ }^{136} \mathrm{Xe}+{ }^{208} \mathrm{~Pb}$ [73], and ${ }^{124} \mathrm{Xe}+{ }^{208} \mathrm{~Pb}$ [73]).

5.1.3. Widths of the velocity spectra In the system ${ }^{136} \mathrm{Xe}_{(1 A \mathrm{GeV})}+p$, the contribution of the multifragmentation mode prevails gradually on the Coulomb-hole mode for elements of larger mass number till, around silicon, the unfolding of the two components becomes too arduous. For silicon nuclides, the contribution of the Coulomb-hole mode is still about half the production cross section of silicon and should still survive for a long range of elements. In this respect, the presence of the Coulomb-hole mode is still appreciable in the widths of the longitudinal-velocity spectra. In fig. 10, the evolution of the mean standard deviation $\left\langle\sigma_{\|}^{\mathrm{p}}\right\rangle$ of the invariant-cross-section distribution is shown as a function of the mass number of the residue for $A>30$. For $A \leq 30$ the mean standard deviation extracted from the forward side of the multifragmentation mode in the invariant-cross-section representation is plotted: this standard deviation is equal to the average standard deviation $\left\langle\sigma_{\|_{i}}^{\mathrm{p}}\right\rangle$ of the components of the multifragmentation mode. Around silicon, the shape of the multifragmentation mode loses its tail and the width extracted from the forward side coincides with the width of the full multifragmentation mode. The mismatch at $A=30$ between the widths of the multifragmentation component and of the full velocity distribution confirms that the contribution of the Coulomb-hole component is still large. 


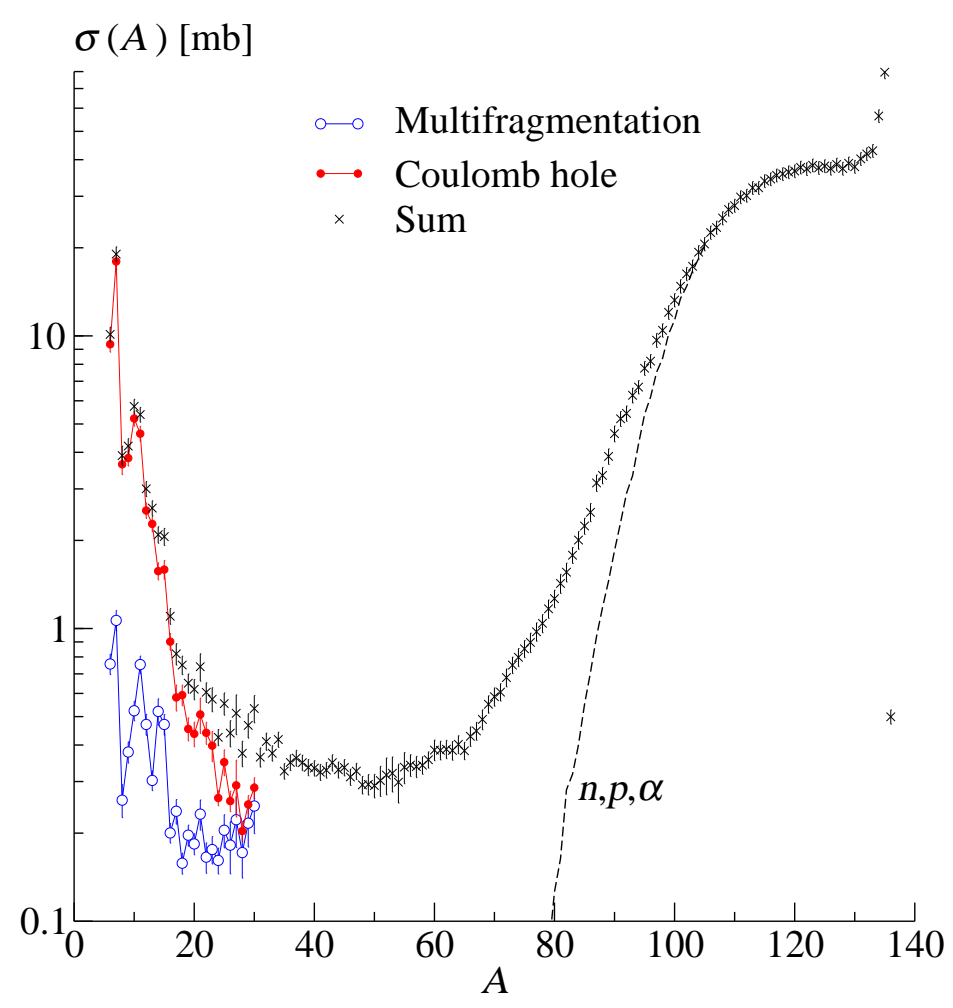

Figure 11. Mass-number distributions of the residue-production cross. For the light fragments the contribution from the two kinematic modes are indicated. A Weisskopf calculation for the expected contribution of the residues which evaporated neutrons, protons and alpha particles is shown.

5.1.4. Nuclide production A survey on the production cross section, taken from ref. [1], is presented in fig. 11, The contributions of the Coulomb-hole and multifragmentation modes, deduced in this work, are indicated; a calculation is also included in the figure to estimate the contribution of a Weisskopf evaporation chain restricted to the emission of only neutrons, protons and alpha particles. Combining the production and the kinematic observables, we can estimate that in the region of mass numbers corresponding to the minimum of cross section, while the information on the Coulomb-hole component gradually vanishes and the production cross section of intermediate-mass fragments drops steeply, the yield of the evaporation residues rises and the kinematics reflected in the velocity spectra is replaced by the characteristic Gaussian shape of the evaporation residues. Above half the mass number of the projectile we observe only the kinematics of the heaviest fragments. When approaching the mass of the projectile, as shown in fig. 10, the velocity width of the invariant cross-section distributions evolves in qualitative agreement with the empirical systematics of Morrissey [60], which is suited for describing the kinematics of heavy residues, and with the prescription of Goldhaber [57].

The production cross section of evaporation residues (in this case we consider any evaporation pattern where intermediate mass fragments are not produced by fission in the first decay steps) varies monotonically as a function of the mass number in agreement 
with the expectation that also the excitation energy of the hot fragments has a monotonic dependence (see fig. 11). In this respect, the fact that the mass distribution of evaporation residues does not extend monotonically towards the lightest masses is an additional indication that intermediate-mass fragments are not fed by evaporation residues. We may remark that this conclusion, clearly suggested by the production cross sections, can not be inferred from the velocity spectra. There is in fact a gradual transition between the shape of the velocity distributions of the heaviest intermediate-mass fragments, which are dominated by the multifragmentation mode and which are almost gaussian, and the gaussian-like spectra of the evaporation residues. For this reason, in lighter systems like ${ }^{56} \mathrm{Fe}+p$ [45], where intermediate-mass fragments and evaporation residues do not occupy distinguishable regions of the production cross-section distribution, we could not identify the transition between the two processes from a similar experimental analysis.

As a result of this section we can deduce the proportions between the different decay modes in the total reaction cross section of the system ${ }^{136} \mathrm{Xe}+p$. In ref. [1] a total reaction cross section of $1393 \pm 72$ [mb] was obtained. We supposed that the Coulomb-hole mode corresponds essentially to binary splits. Although the production of the heavy fragments in the binary splits could not be disentangled, it must be equal to the contribution of the corresponding light partners, which we measured for the greatest part, till $A=30$, where the production cross section already drops to very small values. Under this assumption, the portion of total cross section related to the Coulomb-hole mode could be deduced: we found a value of $59.0 \pm 3.7$ [mb]. The Weisskopf calculation of residues which evaporated protons, neutrons and alpha particles gives an indicative estimation of the corresponding fraction of total cross section of around $90 \%$. The contribution of the multifragmentation mode can not be deduced because neither the fragment multiplicity of the process, nor the total yield of this mode could be deduced from the measurement. The sum of the production cross sections related to the multifragmentation mode which were measured up to silicon is of $8.6 \pm 0.6$ [mb]. By subtracting the measured contribution of the Coulomb-hole mode and the calculated fraction of evaporation residues to the measured total reaction cross section we estimate an indicative cross section of around $35 /\langle M\rangle[\mathrm{mb}]$ for the multifragmentation mode, where $\langle M\rangle$ is the corresponding mean fragment multiplicity, supposed to be larger than two.

5.1.5. Fine structure in the nuclide cross sections A close analysis of the nuclide cross sections reveals a staggering of large magnitude. Such a feature has been observed since long time at different bombarding energies (to indicate part of several contributions, see refs. [74, 75, 76, 77, 78, 79]) The staggering in the nuclide cross sections is particularly evident along chains of nuclides having the same $N-Z$ value. This kind of selection, which was already applied in refs. [80, 81] and employed for the system ${ }^{136} \mathrm{Xe}+p$ in ref. [1], is now presented in fig. 12 for the two decay modes distinctly. The upper row presents the measured cross sections and the lower row quantifies the staggering amplitude according to the procedure introduced by Tracy [82. Despite the smoother 
evolution of the staggering strength for the Coulomb-hole mode, we observe the same general phenomenology for the two modes. In a simple Weisskopf picture this staggering was described as a manifestation of the very last steps of the decay path, independent of the entrance channel; the number of excited levels of the mother nucleus that could decay into the daughter nucleus determines the probability of a channel. In particular, even-mass nuclides show a smooth variation of the separation energy as a function of the element, once shifted by the pairing gap. Therefore, their formation is enhanced due to the pairing gap, which determines a larger number of excited levels of the odd-mass mother nuclei available for the decay into the ground states of even-even daughters rather than odd-odd daughters. This explains the even-odd staggering for $N=Z$ and $N=Z+2$. On the other hand, the formation of odd-mass residues is not determined by the position of their ground states, which are ordered along the same mass parabola for one given mass number. Their formation reflects the structure of the separation energy. Since neutron-rich nuclei have lower separation energy for neutrons, their formation reflects the structure of the neutron separation energy and odd elements are favoured. This explains the reversal of the even-odd staggering for $N=Z+1$ and $N=Z+3$. For the same reason, the staggering becomes less pronounced in the mass-number distribution of cross-sections when more-neutron-rich systems are considered. While chains of nuclides with negative values of $N-Z$ (proton-rich side of the nuclear chart, see refs. [45]) contribute to an even-odd staggering, chains of nuclides with positive values of $N-Z$ contribute to an even-odd staggering only for even values of $N-Z$ (even masses) and contribute to a reversed staggering for odd values of $N-Z$ (odd masses). When dealing with more neutron-rich systems, which present a larger

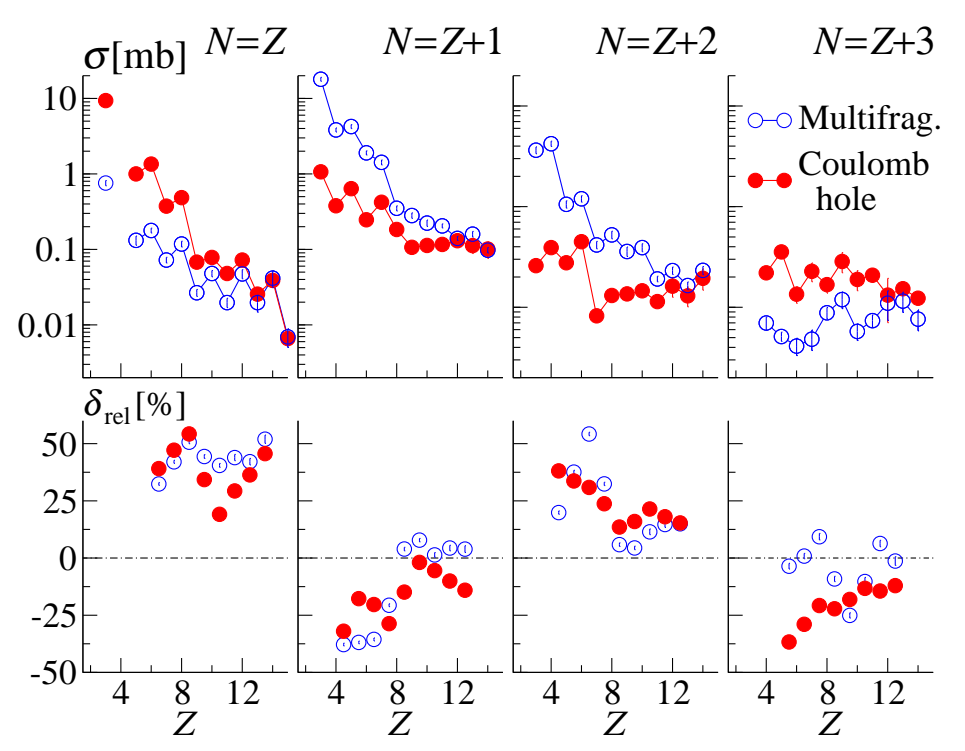

Figure 12. Upper row. Chains of nuclide cross sections with a given value of $N-Z$ present an "even-odd" staggering. Lower row. Amplitude of the staggering analysed by the method of Tracy [82. It is positive when the staggering is "even-odd" and negative when the staggering reverses. 
extension of the nuclide production over neutron-rich isotopes, the sum of the evenodd staggering of the even chains and the reversed staggering of the odd chains lead therefore to a compensation, which is visible in fig. 6. This simple picture is sufficient to conclude from the large amplitude of the staggering that the secondary decay affects largely and in the same manner the production of intermediate mass fragments from both decay modes. We should however remark that a quantitative description of these effects requires first of all a more complete approach, like the Hauser-Feshbach formalism or one of its extensions [83], and the inclusion of Wigner terms in describing the $N=Z$ chain. In addition, it requires the inclusion of decays which go beyond the Hauser-Feshbach approach for compound-nucleus decay, like cluster emission [84, 85]. The study of such a process is of great relevance for describing the formation of the lightest fragments; for a comprehensive review on this subject see refs. [86]

\subsection{Connection between the entrance channel and the exit channel}

The precise knowledge of the mean recoil velocity of the ending fragments of the decay process provides indications on the connection between the exit channel and the entrance channel. Such a study profits from the high-resolution measurement of the inclusive experimental approach. However, this analysis can not be based on single-event observables, due to the large fluctuations induced by the Fermi momenta of abraded nucleons and the recoil due to the decay. In order to smear out these effects, this

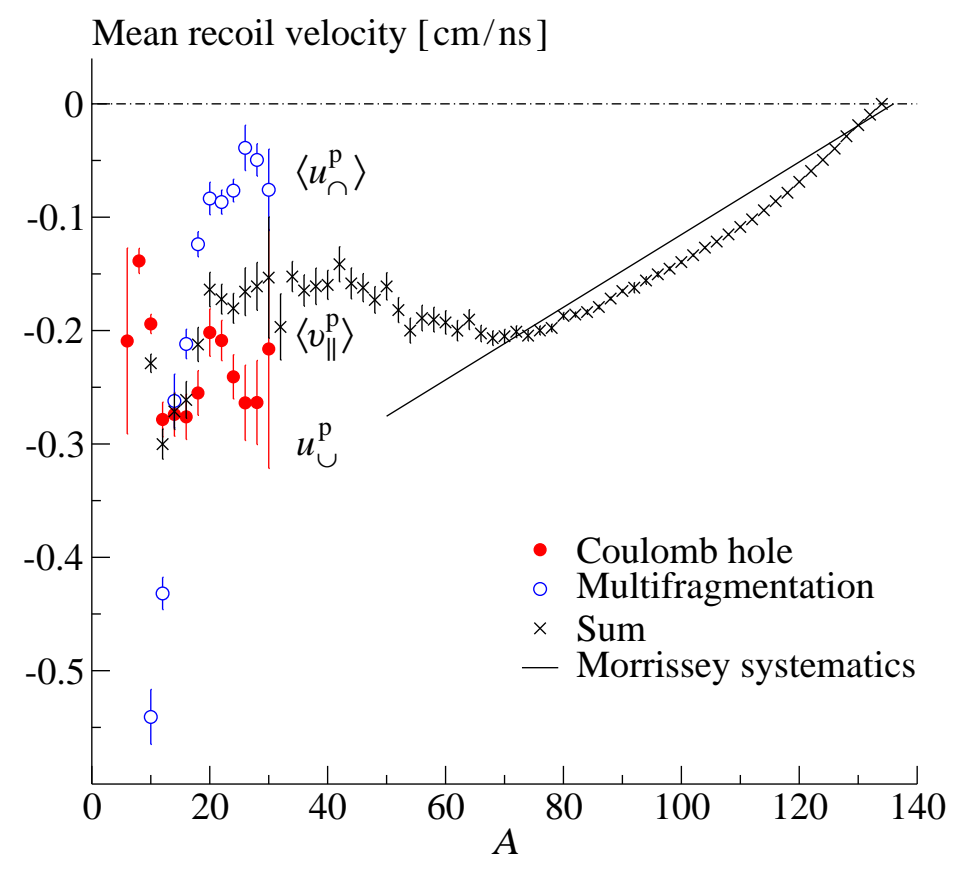

Figure 13. Evolution as a function of the mass of the residue of the mean recoil velocity, deduced for the two kinematic modes separately for lower masses than $A=30$. The mean value of the reconstructed full velocity distribution is shown for the whole mass range. The systematics of Morrissey [60] is shown for comparison. 
analysis is based on employing, as an inclusive quantity, the mean value of the source velocity of one specific component of the velocity distribution in the projectile frame.

It is evident that a study of the entrance channel based on single-event observables, concerning for instance the information on the excitation energy, would go beyond the specificities of this inclusive experimental method. On the other hand, the aim of this study is to join the measurement of the full production, the isotopic identification, and the high resolution of the kinematic observable.

5.2.1. Mean recoil velocities Fig. 2 shows that a systematic displacement in recoil velocity between the two kinematic modes, fission and multifragmentation, could be resolved with high precision since the two components are measured as parts of the same spectrum in the same set of experimental runs. The distinction of the two kinematic modes is a signature of the interplay of two distinct exit channels; their relative displacement, which characterises all spectra of the intermediate-mass fragments shown in fig. 4, attributes the two exit channels to different entrance channels. This displacement is in fact connected to the mean momentum transfer, which reflects the violence of the collision, and which evolves in different ways according to the decay mode. Such study is presented in fig. 13, where three mass regions can be distinguished.

(i) Heavier masses than half the mass of the projectile $(A>68)$. They are necessarily the heaviest fragments produced in the reaction. They cannot be the lighter fragments of a binary decay, and they are not typical multifragmentation products, which should be more than two and should have comparable size.

(ii) Intermediate-mass fragments with $A \leq 30$. Explicit information on the source velocities of the two kinematic modes could be obtained experimentally.

(iii) Fragments in the range $30<A \leq 68$. This is in the minimum of the production as shown in fig. 11, only the average source velocity could be measured, without any kinematic distinction of the decay process.

The region of heavier mass numbers than half the mass of the projectile is dominated by the presence of evaporation residues. Here, the mean recoil $v_{\|}^{\mathrm{p}}$ evolves almost linearly with the mass loss from the projectile velocity down to around $-0.2 \mathrm{~cm} / \mathrm{ns}$ with respect to the projectile frame. The intermediate-mass production attributed to the Coulombhole mode should originate from the decay of heavy residues which evaporate not only nucleons but also heavier fragments. In the process, the accompanying heavy residues should have suffered a large loss of mass. Therefore, it is reasonable that the largest negative mean source velocities, of around $-0.25 \mathrm{~cm} / \mathrm{ns}$, reflected by intermediate-mass fragments issued of binary splits, are close to the source velocities which characterise residues of around half the projectile mass $(A \sim 68)$. With less statistical significance, the data on intermediate-mass fragments from the coulomb-hole mode suggest also the tendency of reducing the mean recoil with increasing mass: the mean recoil varies from around $-0.2 \mathrm{~cm} / \mathrm{ns}$ for $A \sim 5$ to around $-0.25 \mathrm{~cm} / \mathrm{ns}$ for $A \sim 30$, in the projectile frame. This is reasonable, because the emission of heavier intermediate-mass fragments 
from binary decay is expected for higher excitation energies; at the same time, higher excitation energies correspond to smaller impact parameters, reflected by less heavy partners which also tend to have larger negative mean velocities.

Altogether, the experimental results on binary-decay events suggest that the source velocities can be well explained by the empirical systematics of Morrissey [60]: both in the heavy $(A>68)$ and in the light (Coulomb-hole mode measured up to $A=30)$ residues, there is a tendency towards more violent collisions from the heaviest, respectively lightest, masses towards more symmetry. In particular, in the case of binary decays, three quantities are correlated: the evolution of the system towards more symmetric masses is a signal of increasing friction for less peripheral impact parameters, and is reflected in the mean-source velocity. In the case of light intermediate-mass fragments issued of multifragmentation, the correlation between two of these quantities, the size and the impact parameter, is also well known from previous experiments [87], where the fragment size was observed to decrease with increasing energy deposition, for more central collisions.

The mean recoil of the intermediate-mass fragments issued of multifragmentation could be measured in a limited mass range $(A \leq 30)$. For the heaviest fragments, it approaches almost the velocity of the projectile around $A=30$. For the lighter fragments, we find a strong tendency to larger negative values in the projectile frame. Both the velocities of multifragmentation products approaching zero around $A=30$ and the strong trend to very large negative values towards $A \sim 10$ have no reasonable counterpart in the heavy residues. This is not surprising, since, on the one hand, the heavy fragments cannot be produced in a typical multifragmentation process; on the other hand, also the convex pattern in the distribution of invariant cross section of the multifragmentation products contradicts the simultaneous emission of a heavy partner. For larger masses than around $A=15$, the source velocities of the intermediate-mass fragments from multifragmentation variate in the same range of the source velocities of both the heavier and the lighter partners of binary splits; still, the two decay modes can be distinguished by the shape of the velocity distribution.

5.2.2. The mean recoil velocities as a possible indicator of the impact parameter. The new additional information of this experiment, that the size of the intermediate-mass fragments correlates with the mean recoil in the whole mass range and for both the two kinematic modes, makes plausible to establish a further correlation between the mean source velocity and the impact parameter also for the multifragmentation products. We think this is an important generalisation of the interpretation of the kinematic information, which combines results from previous experiments with the new findings of this work. In this respect, this experiment extends the well known Morrissey kinematics which was established for masses very close to the heavy reaction partner, all the way down to the intermediate-mass fragment region. Using the well established correlation of the fragment size in the multifragmentation regime with the impact parameter, we show that the fragment size also correlates with the source velocity. This leads to the 
conclusion that the source velocity is another variable suitable to characterise the impact parameter.

However, differently from the case of the heavy residues, the mean source velocity tagged by the intermediate-mass fragments produced in multifragmentation may manifest a reversed dependence, reducing to very small absolute values in the projectile frame. The experimental disentangling of the two kinetic modes could be extended up to rather heavy fragments: it allowed to make evident the presence of a reversal point of such dependence in the region of the heaviest intermediate mass fragments, around $A=25$, determined by the multifragmentation products. Such finding results from the high-resolution of the inclusive method. For heavier masses, the measurement could no more distinguish the two kinetic modes. However, the reversal is also evident, even if smoothed, by analysing the overall behaviour of the sum of the two kinetic modes, which could be measured for the full range of masses; such observable connects the evolution of the intermediate-mass fragments with the behaviour of the heavy residues. Due to this effect, the impact parameter is not uniquely defined by the mean source velocity in the projectile frame for any fragment size. This ambiguity can be controlled by additionally accounting for the information on the size of the largest fragment: the products which appear with the same mean velocity in the projectile frame are very much separated, if we consider the mass of the heaviest fragment formed in the reaction as an additional criterion. In this respect, a correlation of the mean velocity in the projectile frame with the impact parameter could be established also in the intermediate-mass region.

We may stress that the relation between energy loss and momentum transfer probed by the multifragmentation data should not be regarded within a two-body picture which connects the amount of dissipated energy with the momentum transfer like in dissipative two-body collisions. In this case there would appear a contradiction: multifragmentation products should gain the highest excitation energies, but we observe that the absolute value of their momentum transfer reaches the lowest values. Indeed, this is possible when more than two objects are present in the exit channel. In our experiment there are two objects in the entrance channel, the target proton and the Xe projectile, but many more particles may appear in the exit channel; in this respect, it is possible to fulfil energy and momentum conservation even in cases where the velocity of the Xe-like fragment is almost unchanged and, at the same time, its excitation energy is very high. Such a condition would be associated to an asymmetric emission of cascade or pre-equilibrium particles. Of course, an exclusive experiment could reveal this configuration directly, but at present an inclusive approach has the advantage of giving a more precise measurement of the central region of the longitudinal velocity distribution connected to small angles. Among others, one experimental proof which shows clearly that a many-body configuration in the exit channel can not be described as a twobody kinematics is given by the signature studied in the work [88], where intermediatemass fragments produced in relativistic heavy-ion collisions are predominantly forward emitted. When spallation reactions lead to multifragmentation, the scenario does not change substantially with respect to the heavy-ion collisions. In both cases, a picture 
based on two-body kinematics, dominated by the collision term, and in absence of collective effects, is incomplete. The difference is in the experimental signatures, which are more pronounced in heavy-ion collisions than in spallation. In particular, when proton-induced reactions produce some collective effects, like some thermal pressure created in the cone around the proton traversing the nucleus, these effects are still small in the $1 \mathrm{GeV}$ regime [89].

5.2.3. Possible traces of phase transitions? Supposing that in proton-induced reactions at high incident energies dynamical effects are negligible, we find that the dependence of the recoil observable as a function of the mass number is very different for the Coulombhole and multifragmentation modes. As depicted in fig. 13, for the first mode it varies in a reduced range, while for the second it presents a strong dependence.

Keeping in mind that the recoil observable can be interpreted as an indicator of the impact parameter, this behaviour may be interpreted tentatively as follows. The Coulomb-hole mode is associated to a small range (with respect to the uncertainties) of recoil velocities in the projectile frame: this may indicate that, within the picture of a Liquid-Fission phase transition, this mode is also associated to small energy fluctuations when the system is situated in the coexistence region, and it corresponds to a phase transition with vanishing latent heat, as expected for the Liquid-Fission phase transition [38, 39, 35, 40]. The situation changes completely for the multifragmentation mode, which is associated to a very large range of recoil velocities in the projectile frame; this may correspond to a strong dependence of the fragment configuration on the excitation energy, which corresponds to a finite latent heat. If we consider the heavy residues and the intermediate-mass fragments issued of multifragmentation, and we exclude the Coulomb-hole mode, the plot of fig. 13 gives a good information on the evolution of the recoil as a function of the heaviest fragment. With increasing absolute values of the average recoil in the projectile frame, the system firstly explores a pattern characterised by only heavy evaporation residues, close to the mass of the projectile; it then explores a configuration where both the intermediate-massfragment production and the residues coexist; finally, it evolves towards a situation where the very large average absolute recoil velocity in the projectile frame corresponds only to the multifragmentation mode and no more to the residue production. These inclusive observables are not incompatible with signals of bimodality, which were widely investigated in recent exclusive experiments [26, 27, 28, 29, 30, 31, 32], and which are proposed as a manifestation of the liquid-gas phase transition in nuclear systems. Our interpretation, based on average observables, follows closely this line, but it should be corroborated and quantified by exclusive experiments.

\section{Conclusions and prospects}

In this report we developed a specific procedure for the deconvolution of the distributions of longitudinal velocities of intermediate-mass fragments, which were 
measured inclusively and identified in atomic and mass number. Technically, the experimental approach we adopted in measuring the characteristics of the intermediatemass fragments has several unique advantages: it avoids the contamination from targetlike reaction partners with low kinetic energy which is a difficulty in direct kinematics experiments, and the overloading by the primary beam at small forward angles which challenge inverse-kinematics experiments in absence of a spectrometer; it also profits from the absence of the low-energy detection threshold which characterises silicon detectors, and profits from the absence of magnetic aberrations of the achromatic setting of the small-acceptance spectrometer. As a main disadvantage, the reduced acceptance precludes to measure particle correlations.

As a mayor general result, we could disentangle and analyse quantitatively, in terms of invariant cross sections and in terms of production cross sections two kinematic modes for the exit channel in the spallation of ${ }^{136} \mathrm{Xe}$ at $1 \mathrm{GeV}$ per nucleon, for several isotopes of light elements ranging from lithium to silicon.

One mode is produced by a Coulomb shell in velocity space and results in a convex shape in the distribution of invariant cross section, related to events with low fragment multiplicity and large asymmetry in mass number. This mode is attributed to asymmetric binary decays, and the fission velocity deduced from the measurement is consistent with the binary split of a system close to the mass of the projectile; this is however less true for elements lighter than carbon, which exhibit very high velocities, incompatible with standard calculations. The results are in line with previous similar studies of the formation of intermediate-mass fragments in the lighter system ${ }^{56} \mathrm{Fe}+p$ [45] and in the heavier system ${ }^{238} \mathrm{U}+p[53$ at the same incident energy of $1 \mathrm{GeV}$ per nucleon.

Another mode reflects in a convex shape in the distribution of invariant cross section, related to events with large fragment multiplicity and small asymmetry in mass number. This mode is attributed to multifragmentation. As a relevant result of the present analysis we indicate that, despite the small fraction of the total cross section which feeds multifragmentation, the contribution of this process to single nuclide cross sections is found to be large for intermediate-mass fragments and even dominant for some specific nuclides. Such a large contribution and, more generally, the large production of light elements, may have consequences for technological applications of spallation, such as transmutation and safety.

The main result of this work is the identification of these two decay processes, and the extraction of kinematic and production observables related to the exit channel and associated to the two processes separately. We measured the characteristics of the ending fragments of the decay process, which give valuable information on the heaviest reaction product; the latter is even a fundamental probe of the thermodynamic properties of finite nuclear systems. we also concentrated on the sequence of evaporation decays, reflected in the staggering of nuclide cross sections; such staggering has a comparable amplitude for the two decay modes, and we attributed it to a large fraction of secondary decay; unfortunately, such process has the effect of smearing out the observables which could connect the entrance channel to the exit channel. 
However, signals of this connection are found back in the dependence of the mean recoil as a function of the mass number. By combining results from previous experiments with the new information of this work, we established the inclusive mean source velocity as a valuable indicator of the impact parameter. By relying on such indicator, we firstly confirmed that the Coulomb-hole and the multifragmentation modes, reflect different processes. Then, on the more speculative side, we placed our study in the context of the recent experimental and theoretical investigations on the phase transitions in nuclear systems. We advanced the idea that the two modes could also be related to the phase-transition phenomenology in the finite nuclear system. The Coulomb-hole mode exhibits compatible features with a Liquid-Fission phase transition, characterised by vanishing latent heat. The characteristics which describe the multifragmentation mode are in qualitative accordance with recent observations of a bimodal distributions of the largest fragment charge: for the less violent collisions the multifragmentation mode does not appear and only the heaviest evaporation residues are produced. The multifragmentation mode then gradually prevails for more and more violent collisions till it dominates the decay pattern for the most violent collisions, leading to the production of the smallest residues. This behaviour was found in the study of projectile-like fragments formed in (semi-)peripheral collisions of heavy ions at Fermi energies and it was based on exclusive event selections; it was interpreted as a robust signal of the Liquid-Gas phase transition in the finite nuclear system [26, 27, 28, 29, 30, 31, 32, In this case, the dense phase was identified with residue-like fragments, after the suppression of fission events.

Relativistic nuclear reactions induced by hadrons were already employed for investigating the statistical nature of the decay process [18]. The fraction of incident energy which is transferred to the prefragment appears almost completely as singleparticle excitation, while collective effects like compression are relatively small; for this reason, we suggest that spallation reactions may be also suited for distinguishing the signals of Liquid-Fission and the signals of the Liquid-Gas phase transitions in finite nuclear systems. In the same context, we advance the view that a method of inclusive event selection like the one presented in this paper and, more generally, event selections based on kinematic observables, could be relevant in a further generation of experiments also at Fermi energies, where the measurement of isotopic and kinematic observables in correlation with light-fragments is a mayor request and a foreseen development of the previous-generation exclusive experiments. This is already the direction followed by very recent experiments, where a spectrometer is coupled to a $4 \pi$ detector array [50]. In this respect, high-precision momentum measurements and the nuclide identification of the residues, from inclusive approaches, can offer a further tool of event selection if coupled to correlation measurements, from exclusive approaches. 


\section{Appendix A. Velocity reconstruction with multiple sources}

In this section we give a general description of the deconvolution of the measured longitudinal velocity distributions shown in fig. 2, which leads to the invariant cross sections shown in fig. 4. This procedure is based on the three conditions listed in section 3. For generality, in this section we suppose that one velocity spectrum results from a continuous distribution of sources along the beam axis.

The deconvolution imposes to change among three reference frames, the laboratory frame, the projectile frame, and the reference frame of one given isotropic source, labelled $\mathrm{L}, \mathrm{P}, \mathrm{u}$, respectively. We define the velocity vectors $\boldsymbol{v}^{\mathrm{p}}, \boldsymbol{u}^{\mathrm{p}}$, and $\boldsymbol{v}^{u}=(\boldsymbol{v}-\boldsymbol{u})$, to indicate the velocity of fragments in the projectile frame, the velocity of a given source $u$ in the projectile frame, and the velocity of fragments in the frame of a given source $u$, respectively

We intend to pass by deconvolution from the measured longitudinal velocity distribution $\mathrm{d} I\left(v_{\|}^{\mathrm{p}}\right) / \mathrm{d} v_{\|}^{\mathrm{p}}$, which depends on the acceptance, to the (non-relativistic) invariant cross section $\sigma_{\mathrm{I}}\left(\boldsymbol{v}^{\mathrm{p}}\right)$, which is not depending on the acceptance. Since we must proceed through unfolding over the distribution of sources, we define the corresponding distributions associated to a given source $u$ as in the following scheme:

$\begin{array}{lll} & \text { full space } & \text { source } u \\ \text { acceptance } & \mathrm{d} I\left(v_{\|}^{\mathrm{p}}\right) / \mathrm{d} v_{\|}^{\mathrm{p}} & \mathrm{d}\left[\delta_{u} I\left(v_{\|}^{u}\right)\right] / \mathrm{d} v_{\|}^{u} \\ \text { no acceptance } & \sigma_{\mathrm{I}}\left(\boldsymbol{v}^{\mathrm{p}}\right) & \delta_{u} \sigma_{\mathrm{I}}\left(\boldsymbol{v}^{u}\right)\end{array}$

$\mathrm{d}\left[\delta_{u} I\left(v_{\|}^{u}\right)\right] / \mathrm{d} v_{\|}^{u}$, where $\delta_{u}$ stands for $\mathrm{d} / \mathrm{d} \boldsymbol{u}^{\mathrm{p}}$, is the portion of the measured longitudinal velocity distribution which we attribute to a given source $u$, and $\delta_{u} \sigma_{\mathrm{I}}\left(\boldsymbol{v}^{u}\right)$ is the portion of the invariant cross section which we attribute to the source $u$.

The measured production yield $\mathrm{d} I\left(v_{\|}^{\mathrm{p}}\right) / \mathrm{d} v_{\|}^{\mathrm{p}}$ of one individual nuclide is the integration over the emitting sources of the quantity $\delta_{u} I\left(v_{\|}^{\mathrm{p}}\right)$, which is the portion of the spectrum associated to the sources lying in the interval $\left[u^{\mathrm{p}}, u^{\mathrm{p}}+\mathrm{d} u^{\mathrm{p}}\right]$ :

$$
\frac{\mathrm{d} I\left(v_{\|}^{\mathrm{p}}\right)}{\mathrm{d} v_{\|}^{\mathrm{p}}}=\int_{u^{\mathrm{p}}} \frac{\mathrm{d}}{\mathrm{d} v_{\|}^{\mathrm{p}}} \delta_{u} I\left(v_{\|}^{\mathrm{p}}\right) \mathrm{d} u^{\mathrm{p}} .
$$

The angular acceptance of the magnetic spectrometer, with an almost circular aperture of $\left\langle\alpha^{\mathrm{L}}(\varphi)\right\rangle \approx 15 \mathrm{mr}$ in the laboratory frame (the azimuthal angle $\varphi$ around the beam axis is introduced to take into account the deviation of the acceptance shape from a circle) imposes that a limited selection of the emission-velocity space could be explored and only the selected part of $\delta_{u} I$ appears in the measured yields. The Lorentz transformation attributes to this selected portion a conical boundary along the beam axis in the frame of one emitting source, defined by the velocity vector $\widetilde{\boldsymbol{v}}_{\perp}^{u}$, of magnitude $\widetilde{v}_{\perp}^{u}=\gamma_{\|}^{\mathrm{L}}\left(u^{\mathrm{L}}+v_{\|}^{u}\right) \operatorname{tg} \alpha^{\mathrm{L}}(\varphi)$, and orientation along the azimuthal angle $\varphi$ in the plane orthogonal to the beam axis; $v_{\|}^{u}$ is the longitudinal projection of the boost $\boldsymbol{v}^{u}$ in the frame of the source $u$, imparted to the emitted fragment. By moving to the frame of the source $u$, the contribution $\mathrm{d}\left[\delta_{u} I\left(v_{\|}^{u}\right)\right] / \mathrm{d} v_{\|}^{u}$ of the source $u$ to the measured yields is 
described by the integration of the transverse velocity $v_{\perp}^{u}$ over the portion of the plane selected by the ensemble of vectors $\widetilde{\boldsymbol{v}}_{\perp}^{u}$, so that

$$
\frac{\mathrm{d}\left[\delta_{u} I\left(v_{\|}^{u}\right)\right]}{\mathrm{d} v_{\|}^{u}}=\int_{0}^{2 \pi} \int_{0}^{\left|\widetilde{v}_{\perp}^{u}\right|} v_{\perp}^{u} \frac{\mathrm{d}\left[\delta_{u} \sigma\left(\boldsymbol{v}^{u}\right)\right]}{\mathrm{d} \boldsymbol{v}^{u}} \mathrm{~d} v_{\perp}^{u} \mathrm{~d} \varphi,
$$

where $\sigma$ is the production cross section for the nuclide associated to the velocity spectrum. Since in our case the velocities in the source frame are not relativistic, the argument of the integral in eq. A.2 is equivalent to the invariant cross section as a function of the velocity in the source frame:

$$
\frac{\mathrm{d}\left[\delta_{u} \sigma\left(\boldsymbol{v}^{u}\right)\right]}{\mathrm{d} \boldsymbol{v}^{u}}=\frac{\mathrm{m}^{2}}{\mathrm{c}^{2}} E^{u} \frac{\mathrm{d}\left[\delta_{u} \sigma\left(\boldsymbol{v}^{u}\right)\right]}{\mathrm{d} \boldsymbol{p}^{u}}=\frac{\mathrm{m}^{2}}{\mathrm{c}^{2}} \delta_{u} \sigma_{\mathrm{I}}\left(\boldsymbol{v}^{u}\right),
$$

where $E^{u}$ and $\boldsymbol{p}^{u}$ indicate the total energy and the momentum in the source frame, respectively. Introducing the invariant cross section in the integral we write:

$$
\frac{\mathrm{d}\left[\delta_{u} I\left(v_{\|}^{u}\right)\right]}{\mathrm{d} v_{\|}^{u}}=\frac{\mathrm{m}^{2}}{\mathrm{c}^{2}} \int_{0}^{2 \pi} \int_{0}^{\left|\widetilde{v}_{\perp}^{u}\right|} \delta_{u} \sigma_{\mathrm{I}}\left(\boldsymbol{v}^{u}\right) v_{\perp}^{u} \mathrm{~d} v_{\perp}^{u} \mathrm{~d} \varphi,
$$

This expression does not impose any constraint for the distribution of emitted fragments. Under the assumption of isotropy $\delta_{u} \sigma_{\mathrm{I}}\left(\boldsymbol{v}^{u}\right)=\delta_{u} \sigma_{\mathrm{I}}\left(v^{u}\right)=\left[1 / 4 \pi\left(v^{u}\right)^{2}\right] \mathrm{d}\left[\delta_{u} \sigma\left(v^{u}\right)\right] / \mathrm{d} v^{u}$ and by substituting the integration variable $v_{\perp}^{u}$ with $v^{u}=\sqrt{\left(v_{\|}^{u}\right)^{2}+\left(v_{\perp}^{u}\right)^{2}}$ we can write (indicating $\boldsymbol{v}_{\|}^{u}$ the vector of magnitude $v_{\|}^{u}$, oriented along the beam direction):

$$
\frac{\mathrm{d} I\left(v_{\|}^{\mathrm{p}}\right)}{\mathrm{d} v_{\|}^{\mathrm{p}}}=\frac{\mathrm{m}^{2}}{\mathrm{c}^{2}} \int_{u^{\mathrm{p}}} \int_{0}^{2 \pi} \int_{\left|v_{\|}^{u}\right|}^{\left|v^{u}\right|} \delta_{u} \sigma_{\mathrm{I}}\left(v^{u}\right) v^{u} \mathrm{~d} v^{u} \mathrm{~d} \varphi \mathrm{d} u^{\mathrm{p}},
$$

where the integral boundaries are $\left|v_{\|}^{u}\right|=\left|v_{\|}^{\mathrm{p}}-u^{\mathrm{p}}\right|$ and $\left|v^{u}\right|=\sqrt{\left(v_{\|}^{\mathrm{p}}-u^{\mathrm{p}}\right)^{2}+\left(\widetilde{v}_{\perp}^{u}\right)^{2}}$.

The distribution of invariant cross section $\sigma_{\mathrm{I}}\left(v^{u}\right)$, presented in fig. 目 was deduced by the deconvolution of the integral eq. (A.5). The deconvolution can be solved only by restricting to a finite number of degrees of freedom: this implies that the number of sources is limited (the integral over $u^{\mathrm{p}}$ is converted into a discrete sum over the sources). With this general prescription we constructed an axial-symmetric distribution of invariant cross section in the beam frame $\sigma_{\mathrm{I}}\left(v_{\|}^{\mathrm{p}}\right)$ as the superposition of isotropic emission patterns, each one defined by one individual source:

$$
\frac{\mathrm{c}^{2}}{\mathrm{~m}^{2}} \frac{\mathrm{d} \sigma}{\mathrm{d} \boldsymbol{v}^{\mathrm{p}}}=\sigma_{\mathrm{I}}\left(\boldsymbol{v}^{\mathrm{p}}\right)=\int_{u^{\mathrm{p}}} \delta_{u} \sigma_{\mathrm{I} u}\left(\left|\boldsymbol{v}^{\mathrm{p}}-\boldsymbol{u}^{\mathrm{p}}\right|\right) \mathrm{d} u^{\mathrm{p}} .
$$

This spectrum would be obtained directly from the experimental measurement only if two conditions were satisfied. First, the angular acceptance should not impose any cut. Second, also the transversal components of the velocity should be measured. 


\section{Appendix B. Parameters of the invariant cross sections}

The analysis described in this work results from the deconvolution procedure introduced in section 3 and applied to the analysis of section 4, so as to produce the results sketched in fig. 3. As an outcome, the procedure gave the parameters listed in table B1.

Table B1. Parameters of the invariant cross sections, which determine the plots in fig. 4 fig. 10 and fig. 13 The parameters are the mean recoil of the Coulombhole mode $u_{\llcorner}^{\mathrm{p}}$, the ridge of the ring associated to the Coulomb-hole mode in the invariant cross section distribution $v_{\text {peak }}$, the standard deviation of the Coulombhole mode in the invariant cross section distribution $\sigma_{\| \bullet}^{\mathrm{p}}$, the mean recoil of the multifragmentation mode $\left\langle u_{\sim}^{\mathrm{P}}\right\rangle$, and the average standard deviation of the components of the multifragmentation mode in the invariant cross section distribution $\left\langle\sigma_{\| \sim}^{\mathrm{p}}\right\rangle$.

\begin{tabular}{|c|c|c|c|c|c|}
\hline \multirow[t]{2}{*}{$A$} & $u_{\smile}^{\mathrm{p}}$ & $v_{\text {peak }}$ & & \multicolumn{2}{|c|}{$\left\langle u_{\curvearrowleft}^{\mathrm{p}}\right\rangle \quad\left\langle\sigma_{\| \curvearrowright}^{\mathrm{p}}\right\rangle$} \\
\hline & \multicolumn{5}{|l|}{$\mathrm{Li}$} \\
\hline 6 & $-0.21(11)$ & $2.49(21)$ & $0.497(43)$ & $-1.04(14)$ & $0.90(12)$ \\
\hline 7 & $-0.21(11)$ & $2.37(21)$ & $0.471(43)$ & $-1.26(14)$ & $0.87(12)$ \\
\hline \multirow[t]{2}{*}{8} & $-0.091(21)$ & $2.339(40)$ & $0.433(45)$ & $-0.749(62)$ & $0.930(23)$ \\
\hline & \multicolumn{5}{|c|}{$\mathrm{Be}$} \\
\hline 9 & $-0.1839(74)$ & $2.292(14)$ & $0.448(11)$ & $-0.666(21)$ & $0.836(21)$ \\
\hline 10 & $-0.1018(99)$ & $2.170(18)$ & $0.454(18)$ & $-0.636(21)$ & $0.850(21)$ \\
\hline 11 & $-0.052(21)$ & $2.083(39)$ & $0.366(42)$ & $-0.329(73)$ & $1.286(32)$ \\
\hline \multirow[t]{2}{*}{12} & $-0.024(28)$ & $2.107(51)$ & $0.427(44)$ & $-0.270(32)$ & $0.826(21)$ \\
\hline & \multicolumn{5}{|l|}{$\mathrm{B}$} \\
\hline 10 & $-0.389(23)$ & $2.270(43)$ & $0.498(38)$ & $-0.25(13)$ & $0.890(22)$ \\
\hline 11 & $-0.2405(98)$ & $2.180(18)$ & $0.462(14)$ & $-0.564(21)$ & $0.821(21)$ \\
\hline 12 & $-0.316(23)$ & $1.985(43)$ & $0.478(32)$ & $-0.485(23)$ & $0.918(23)$ \\
\hline \multirow[t]{2}{*}{13} & $-0.245(80)$ & $2.10(15)$ & $0.45(13)$ & $-0.567(18)$ & $0.702(18)$ \\
\hline & \multicolumn{5}{|l|}{$\mathrm{C}$} \\
\hline 11 & $-0.372(36)$ & $2.361(67)$ & $0.579(87)$ & $-0.571(50)$ & $1.07(11)$ \\
\hline 12 & $-0.315(12)$ & $2.108(21)$ & $0.457(17)$ & $-0.438(18)$ & $0.682(17)$ \\
\hline 13 & $-0.256(15)$ & $2.052(28)$ & $0.451(21)$ & $-0.352(16)$ & $0.633(16)$ \\
\hline 14 & $-0.310(18)$ & $1.944(34)$ & $0.424(24)$ & $-0.294(25)$ & $0.984(25)$ \\
\hline \multirow[t]{2}{*}{15} & $-0.190(76)$ & $1.98(14)$ & $0.41(13)$ & $-0.325(87)$ & $0.850(78)$ \\
\hline & \multicolumn{5}{|l|}{$\mathrm{N}$} \\
\hline 13 & $-0.187(55)$ & $2.17(10)$ & $0.457(95)$ & $-0.291(19)$ & $0.749(19)$ \\
\hline 14 & $-0.297(29)$ & $2.063(53)$ & $0.470(72)$ & $-0.501(27)$ & $0.631(37)$ \\
\hline 15 & $-0.248(26)$ & $1.997(47)$ & $0.396(30)$ & $-0.181(37)$ & $0.680(22)$ \\
\hline 16 & $-0.257(29)$ & $1.908(53)$ & $0.384(45)$ & $-0.055(14)$ & $0.546(14)$ \\
\hline 17 & $-0.177(74)$ & $1.76(13)$ & $0.424(83)$ & $-0.274(12)$ & $0.470(21)$ \\
\hline
\end{tabular}




\begin{tabular}{|c|c|c|c|c|c|}
\hline \multirow[t]{2}{*}{$A$} & $u_{\smile}^{\mathrm{p}}$ & \multirow[t]{2}{*}{$v_{\text {peak }}$} & \multirow[t]{2}{*}{$\sigma_{\| \smile}^{\mathrm{p}}$} & \multicolumn{2}{|c|}{$\left\langle u_{\curvearrowright}^{\mathrm{p}}\right\rangle \quad\left\langle\sigma_{\| \curvearrowright}^{\mathrm{p}}\right\rangle$} \\
\hline & $\mathrm{O}$ & & & & \\
\hline 15 & $-0.16(13)$ & $2.06(27)$ & $0.62(21)$ & $-0.235(33)$ & $0.604(74)$ \\
\hline 16 & $-0.342(18)$ & $1.921(32)$ & $0.425(33)$ & $-0.359(15)$ & $0.604(15)$ \\
\hline 17 & $-0.273(11)$ & $1.913(19)$ & $0.373(23)$ & $-0.171(21)$ & $0.845(21)$ \\
\hline 18 & $-0.241(17)$ & $1.859(31)$ & $0.366(24)$ & $-0.130(13)$ & $0.518(13)$ \\
\hline \multirow[t]{2}{*}{19} & $-0.290(43)$ & $1.829(79)$ & $0.384(54)$ & $-0.165(19)$ & $0.729(18)$ \\
\hline & $\mathrm{F}$ & & & & \\
\hline 17 & $-0.204(62)$ & $2.04(11)$ & $0.265(93)$ & $-0.222(80)$ & $0.917(44)$ \\
\hline 18 & $-0.329(22)$ & $1.843(40)$ & $0.382(40)$ & $-0.161(16)$ & $0.633(16)$ \\
\hline 19 & $-0.240(36)$ & $1.881(66)$ & $0.351(66)$ & $-0.072(14)$ & $0.566(17)$ \\
\hline 20 & $-0.262(20)$ & $1.805(37)$ & $0.338(35)$ & $-0.014(14)$ & $0.562(14)$ \\
\hline \multirow[t]{2}{*}{21} & $-0.125(47)$ & $1.779(96)$ & $0.413(68)$ & $-0.127(32)$ & $0.631(45)$ \\
\hline & $\mathrm{Ne}$ & & & & \\
\hline 19 & $-0.373(95)$ & $1.87(17)$ & $0.37(15)$ & $-0.189(84)$ & $0.578(60)$ \\
\hline 20 & $-0.222(31)$ & $1.853(56)$ & $0.353(63)$ & $-0.220(15)$ & $0.612(15)$ \\
\hline 21 & $-0.196(16)$ & $1.831(30)$ & $0.360(35)$ & $-0.064(13)$ & $0.532(13)$ \\
\hline 22 & $-0.159(17)$ & $1.788(30)$ & $0.339(24)$ & $-0.021(12)$ & $0.460(12)$ \\
\hline \multirow[t]{2}{*}{23} & $-0.246(55)$ & $1.688(96)$ & $0.367(75)$ & $-0.124(27)$ & $0.498(18)$ \\
\hline & $\mathrm{Na}$ & & & & \\
\hline 22 & $-0.231(30)$ & $1.782(55)$ & $0.373(54)$ & $-0.119(12)$ & $0.479(12)$ \\
\hline 23 & $-0.2661(83)$ & $1.730(15)$ & $0.330(19)$ & $-0.144(12)$ & $0.486(12)$ \\
\hline 24 & $-0.240(10)$ & $1.712(18)$ & $0.315(21)$ & $-0.081(12)$ & $0.495(12)$ \\
\hline \multirow[t]{2}{*}{25} & $-0.186(32)$ & $1.647(58)$ & $0.330(42)$ & $-0.083(10)$ & $0.400(12)$ \\
\hline & $\mathrm{Mg}$ & & & & \\
\hline 23 & $-0.14(10)$ & $1.893(69)$ & $0.33(14)$ & $-0.141(69)$ & $0.553(28)$ \\
\hline 24 & $-0.245(56)$ & $1.675(96)$ & $0.387(87)$ & $-0.029(14)$ & $0.567(14)$ \\
\hline 25 & $-0.323(20)$ & $1.587(34)$ & $0.342(36)$ & $-0.086(17)$ & $0.666(17)$ \\
\hline 26 & $-0.242(21)$ & $1.611(37)$ & $0.342(44)$ & $-0.002(14)$ & $0.556(14)$ \\
\hline \multirow[t]{2}{*}{27} & $-0.316(79)$ & $1.51(13)$ & $0.31(11)$ & $-0.108(54)$ & $0.690(37)$ \\
\hline & $\mathrm{Al}$ & & & & \\
\hline 25 & $-0.18(78)$ & $1.61(14)$ & $0.39(19)$ & $0.03(31)$ & $0.39(98)$ \\
\hline 26 & $-0.226(71)$ & $1.60(12)$ & $0.367(91)$ & $-0.079(20)$ & $0.610(22)$ \\
\hline 27 & $-0.258(41)$ & $1.538(68)$ & $0.379(83)$ & $-0.017(15)$ & $0.586(15)$ \\
\hline 28 & $-0.284(24)$ & $1.542(41)$ & $0.332(31)$ & $-0.045(15)$ & $0.598(15)$ \\
\hline 29 & $-0.194(56)$ & $1.497(94)$ & $0.31(14)$ & $-0.073(28)$ & $0.575(38)$ \\
\hline 30 & $-0.330(98)$ & $1.47(16)$ & $0.30(10)$ & $-0.08(16)$ & $0.667(85)$ \\
\hline
\end{tabular}




\begin{tabular}{llllll}
$A$ & \multicolumn{1}{c}{$u_{\smile}^{\mathrm{p}}$} & \multicolumn{1}{c}{$v_{\text {peak }}$} & $\sigma_{\| \smile}^{\mathrm{p}}$ & $\left\langle u_{\wedge}^{\mathrm{p}}\right\rangle$ & $\left\langle\sigma_{\| \wedge}^{\mathrm{p}}\right\rangle$ \\
\hline & $\mathrm{Si}$ & & & & \\
28 & $-0.274(38)$ & $1.493(63)$ & $0.374(60)$ & $-0.086(16)$ & $0.659(16)$ \\
29 & $-0.333(75)$ & $1.42(12)$ & $0.373(75)$ & $-0.014(16)$ & $0.633(16)$ \\
30 & $-0.19(16)$ & $1.56(26)$ & $0.39(19)$ & $-0.076(16)$ & $0.632(81)$ \\
31 & $-0.213(33)$ & $1.460(54)$ & $0.295(55)$ & $-0.077(11)$ & $0.442(11)$ \\
32 & $-0.22(17)$ & $1.42(27)$ & $0.31(15)$ & $-0.08(24)$ & $0.61(25)$
\end{tabular}

\section{References}

[1] P. Napolitani, K.-H. Schmidt, L. Tassan-Got, P. Armbruster, T. Enqvist, A. Heinz, V. Henzl, D. Henzlova, A. Kelić, R. Pleskač, M.V. Ricciardi, C. Schmitt, O. Yordanov, L. Audouin, M. Bernas, A. Lafriaskh, F. Rejmund, C. Stéphan, J. Benlliure, E. Casarejos, M. Fernandez Ordonez, J. Pereira, A. Boudard, B. Fernandez, S. Leray, C. Villagrasa and C. Volant, Phys. Rev. C 76, 064609 (2007).

[2] J. Hüfner Phys. Rep. 125, 129 (1985).

[3] W.G.Lynch, Annual Review of Nucl. and Part. Science 37, 493 (1987).

[4] J. Robb Grover, Phys. Rev. 126, 1540 (1962).

[5] S.B. Kaufman, M.W. Weisfield, E.P. Steinberg, B.D. Wilkins, D. Henderson, Phys. Rev. C 14, 1121 (1976).

[6] A.I. Warwick, A. Baden, H.H. Gutbrod, M.R. Maier, J. Péter, H.G. Ritter, H. Stelzer, H.H. Wieman, F. Weik, M. Freedman, D.J. Henderson, S.B. Kaufman, E.P. Steinberg and B.D. Wilkins, Phys. Rev. Lett. 48, 1719 (1982).

[7] A.S. Hirsch, A. Bujak, J.E. Finn, L.J. Gutay, R.W. Minich, N.T. Porile, R.P. Scharenberg, B.C. Stringfellow, and F. Turkot, Phys. Rev. C 29, 508 (1984).

[8] L.N. Andronenko, A.A. Kotov, L.A. Vaishnene, W. Neubert, H.W. Barz, J.P. Bondorf, R. Donangelo and H. Schulz, Phys. Lett. B174, 18 (1986).

[9] H.W. Barz, J.P. Bondorf, H. Schulz, L.N. Andronenko, A.A. Kotov, L.A. Vaishnene and W. Neubert, Nucl. Phys. A460, 714 (1986).

[10] A.A. Kotov, L.N. Andronenko, M.N. Andronenko, Y.I. Gusev, K.V. Lukashin, W. Neubert, D.M. Seliverstov, I.I. Strakovsky and L.A. Vaishnene, Nucl. Phys. A583, 575 (1995).

[11] W.-C. Hsi, K. Kwiatkowski, G. Wang, D.S. Bracken, E. Cornell, D.S. Ginger, V.E. Viola, N.R. Yoder, R.G. Korteling, F. Gimeno-Nogures, E. Ramakrishnan, D. Rowland, S.J. Yennello, M.J. Huang, W.G. Lynch, M.B. Tsang, H. Xi, Y.Y. Chu, S. Gushue, L.P. Remsberg, K.B. Morley, and H. Breuer, Phys. Rev. Lett. 79, 817 (1997).

[12] S.P. Avdeyev, V.A. Karnaukhov, W.D. Kuznetsov, L.A. Petrov, V.K. Rodionov, A.S. Zubkevich, H. Oeschler, O.V. Bochkarev, L.V. Chulkov, E.A. Kuzmin, A. Budzanovski, W. Karcz, M. Janicki, E. Norbeck, A.S. Botvina, W.A. Friedman, W. Nörenberg and G. Papp, Eur. Phys. J. A3, 75 (1998).

[13] S.J. Sanders, A. Szanto de Toledo , C. Beck, Phys. Rep. 311, 487 (1999).

[14] L.G. Moretto, Nucl. Phys. A247, 211 (1975).

[15] L.G. Moretto and G.J. Wozniak, Pramana, J. Phys. 33, 209 (1989)

[16] Statistical Models for Nuclear Decay: From Evaporation to Vaporization, A.J. Cole Fundamental and Applied Nuclear Phyisics Series (IoP), Edt. R.R. Betts and W. Greiner. (2000)

[17] Topical Volume: Dynamics and Thermodynamics with Nuclear Degrees of Freedom, Eur. Phys. J. A 30 (1) (2006).

[18] V.E. Viola, K. Kwiatkowski, L. Beaulieu, D.S. Bracken, H. Breuer, J. Brzychczyk, R.T. de Souza, D.S. Ginger, W-C. Hsi, R.G. Korteling, T. Lefort, W.G. Lynch, K.B. Morley, R. Legrain, L. 
Pienkowski, .C. Pollacco, E. Renshaw, A. Ruangma, M.B. Tsang, C. Volant, G. Wang, S.J. Yennello and N.R. Yoder, Phys. Rep. 434, 1 (2006).

[19] V.A. Karnaukhov, S.P. Avdeyev, W.D. Kuznetsov, L.A. Petrov, V.K. Rodionov, A.S. Zubkevich, H. Oeschler, O.V. Bochkarev, L.V. Chulkov, E.A. Kuzmin, A. Budzanowski, W. Karcz, M. Janicki, E. Norbeck and A.S. Botvina, Yad.Fiz. 62, No 2, 2272 (1999); Phys. Atomic Nuclei 62, 237 (1999).

[20] V.A. Karnaukhov, S.P. Avdeyev, E.V. Duginova, L.A. Petrov, V.K. Rodionov, H. Oeschler, A. Budzanowski, W. Karcz, M. Janicki, O.V. Bochkarev, E.A. Kuzmin, L.V. Chulkov, E. Norbeck and A.S. Botvina, Yad.Fiz. 66, 1282 (2003); Phys.Atomic Nuclei 66, 1242 (2003).

[21] L.G. Moretto and G.J. Wozniak, Prog. Part. Nucl. Phys. 21, 401 (1988).

[22] J.A. Lopez and J.Randrup, Nucl. Phys. A571, 379 (1994).

[23] J.P. Bondorf, A.S. Botvina, A.S. Iljinov, I.N. Mishustin and K. Sneppen, Phys. Rep. 257, 133 (1995).

[24] Al.H. Raduta and Ad.R. Raduta, Phys. Rev. C 55, 1344 (1997).

[25] D.H.E. Gross, Microcanonical Thermodynamics: Phase Transitions in Small Systems, Lecture Notes in Physics 66, (World Scientific Singapore, 2001).

[26] G. Tăbăcaru, B. Borderie, P. Désesquelles, M. Pârlog, M.-F. Rivet, R. Bougault, B. Bouriquet, A.M. Buta, E. Galichet, B. Guiot, P. Lautesse, N. Le Neindre, L. Manduci, E. Rosato, B. Tamain, M. Vigilante, and J.P. Wieleczko, Eur. Phys. J. A18 (2003) 103.

[27] O. Lopez and M.-F. Rivet, Eur. Phys. J. A30, 263 (2006).

[28] M. Pichon, B. Tamain, R. Bougault, F. Gulminelli, O. Lopez, E. Bonnet, B. Borderie, A. Chbihi, R. Dayras, J.D. Frankland, E. Galichet, D. Guinet, P. Lautesse, N. Le Neindre, M. Pârlog, M.-F. Rivet, R. Roy, E. Rosato, E. Vient, M. Vigilante, C. Volant, J.P. Wieleczko and B. Zwieglinski, Nucl. Phys. A779, 267 (2006).

[29] E. Bonnet, F. Gulminelli, B. Borderie, N. Le Neindre, M.-F. Rivet, Proceedings of the XLV International Winter Meeting on Nuclear Physics (2007) 1, arXiv:0704.1396

[30] E. Bonnet, B. Borderie, N. Le Neindre and M.F. Rivet International Workshop on Multifragmentation and Related Topics (IWM2007), Caen (2007), arXiv:0802.0415.

[31] E. Bonnet, D. Mercier, Bernard Borderie, F. Gulminelli, M.-F. Rivet, B. Tamain, R. Bougault, A. Chbihi, R. Dayras, J.D. Frankland, E. Galichet, F. Gagnon-Moisan, D. Guinet, P. Lautesse, J. Lukasik, N. Le Neindre, M. Pârlog, E. Rosato, R. Roy, M. Vigilante, J.P. Wieleczko and B. Zwieglinski, Phys. Rev. Lett. 103, 072701 (2009).

[32] M.Bruno, F.Gulminelli, F.Cannata, M.D'Agostino, F.Gramegna and G.Vannini, Nucl. Phys. A807, 48 (2008).

[33] F. Gulminelli and Ph. Chomaz, Phys. Rev. Lett. 82, 1402 (1999).

[34] Ph. Chomaz and F. Gulminelli, in Dynamics and Thermodynamics of systems with long range interactions, Lecture Notes in Physics vol.602, (Springer, 2002).

[35] F. Gulminelli, Ph. Chomaz, Al.H. Raduta and Ad.R. Raduta, Phys. Rev. Lett. 91, 202701 (2003).

[36] O. Lopez, D.Lacroix and E. Vient, Phys. Rev. Lett. 95, 242701 (2005).

[37] F. Gulminelli, Nucl. Phys. A791, 165 (2007).

[38] G. Lehaut, F. Gulminelli and O. Lopez, Phys. Rev. Lett. 102, 142503 (2009).

[39] F. Gulminelli and G. Lehaut, Int. J. Mod. Phys. E18, 1022 (2009).

[40] G. Chaudhuri, S. Das Gupta and F. Gulminelli, Nucl. Phys. A815, 89 (2009).

[41] T. Enqvist, J. Benlliure, F. Farget, K.-H. Schmidt, P. Armbruster, M. Bernas, L. Tassan-Got, A. Boudard, R. Legrain, C. Volant, C. Boeckstiegel, M. de Jong and J. P. Dufour, Nucl. Phys. A658, 47 (1999).

[42] M. Bernas, P. Armbruster, J. Benlliure, A. Boudard, E. Casarejos, S. Czajkowski, T. Enqvist, R. Legrain, S. Leray, B. Mustapha, P. Napolitani, J. Pereira-Conca, F. Rejmund, M.V. Ricciardi, K.-H. Schmidt, C. Stéphan, J. Taieb, L. Tassan-Got and C. Volant, Nucl. Phys. A725, 213 (2003).

[43] H. Geissel, P. Armbruster, K.H. Behr, A. Brünle, K. Burkard, M. Chen, H. Folger, B. Franczak, H. 
Keller, O. Klepper, B. Langenbeck, F. Nickel, E. Pfeng, M. Pfützner, E. Roeckl, K. Rykaczewski, I. Schall, D. Schardt, C. Scheidenberger, K.-H. Schmidt, A. Schroter, T. Schwab, K. Sümmerer, M. Weber, G. Münzenberg, T. Brohm, H.-G. Clerc, M. Fauerbach, J.-J. Gaimard, A. Grewe, E. Hanelt, B. Knödler, M. Steiner, B. Voss, J. Weckenmann, C. Ziegler, A. Magel, H. Wollnik, J.P. Dufour, Y. Fujita, D.J. Vieira and B. Sherrill, Nucl. Instrum. Methods B70, 286 (1992).

[44] K-H. Schmidt, E. Hanelt, H. Geissel, G. Münzenberg and J.-P. Dufour, Nucl. Instr. Meth. A260, 287 (1987).

[45] P. Napolitani, K.-H. Schmidt, A.S. Botvina, F. Rejmund, L. Tassan-Got and C.Villagrasa, Phys. Rev. C 70, 054607 (2004).

[46] C. Villagrasa-Canton, A. Boudard, J.-E. Ducret, B. Fernandez, S. Leray, C. Volant, P. Armbruster, T. Enqvist, F. Hammache, K. Helariutta, B. Jurado, M.V. Ricciardi, K.-H. Schmidt, K. Sümmerer, F. Vivès, O. Yordanov, L. Audouin, C.-O. Bacri, L. Ferrant, P. Napolitani, F. Rejmund, C. Stéphan, L. Tassan-Got, J. Benlliure, E. Casarejos, M. Fernandez-Ordoñez, J. Pereira, S. Czajkowski, D. Karamanis, M. Pravikoff, J.S. George, R.A. Mewaldt, N. Yanasak, M. Wiedenbeck, J.J. Connell, T. Faestermann, A. Heinz and A. Junghans, Phys. Rev. C 75, 044603 (2007).

[47] E. Le Gentil, T. Aumann, C.O. Bacri, J. Benlliure, S. Bianchin, M. Böhmer, A. Boudard, J. Brzychczyk, E. Casarejos, M. Combet, L. Donadille, J.E. Ducret, M. Fernandez-Ordoñez, R. Gernhäuser, H. Johansson, K. Kezzar, T. Kurtukian-Nieto, A. Lafriakh, F. Lavaud, A. Le Fèvre, S. Leray, J. Lühning, J. Lukasik, U. Lynen, W.F.J. Müller, P. Pawlowski, S. Pietri, F. Rejmund, C. Schwarz, C. Sfienti, H. Simon, W. Trautmann, C. Volant, and O. Yordanov Phys. Rev. Lett. 100, 022701 (2008).

[48] U.L. Businaro and S. Gallone, Nuovo Cimento 1, 629 (1955).

[49] U.L. Businaro and S. Gallone, Nuovo Cimento 1, 1277 (1955).

[50] A. Chbihi, Proceedings of the IWM07 International Workshop on Multifragmentation and Related Topics, Caen, France, 2007, edited by B. Borderie et al., Conf. Proc. Vol. 95 (Italian Physical Society, Bologna, 2008), p. 277.

[51] K.K. Gudima, S.G. Mashnik, and V.D. Toneev, Nucl. Phys. A401, 329 (1983).

[52] J. Benlliure, J. Pereira-Conca, K.-H. Schmidt, Nucl. Instrum. Methods. A478, 493 (2002).

[53] M. V. Ricciardi, P. Armbruster, J. Benlliure, M. Bernas, A. Boudard, S. Czajkowski, T. Enqvist, A. Kelic, S. Leray, R. Legrain, B. Mustapha, J. Pereira,F. Rejmund, K. -H. Schmidt, C. Stéphan,

L. Tassan-Got, C. Volant and O. Yordanov, Phys. Rev. C 73, 014607 (2006).

[54] S. Pullanhiotan, M. Rejmund, A. Navin, W. Mittig, S. Bhattacharyya, Nucl. Instrum. Methods. A593, 343 (2008).

[55] S. Pullanhiotan, A. Chatterjee, B. Jacquot, A. Navin, M. Rejmund, Nucl. Instrum. Methods. B266, 4148 (2008).

[56] R. Serber, Phys. Rev. 72, 1114 (1947).

[57] A.S. Goldhaber, Phys. Lett. B53, 306 (1974).

[58] A.S. Botvina, A.S. Iljinov and I.N. Mishustin, JETP Lett. 42, 572 (1985).

[59] A.S. Botvina, A.S. Iljinov and I.N. Mishustin, Nucl. Phys. A507, 649 (1990).

[60] D.J. Morrissey, Phys. Rev. C 39, 460 (1989).

[61] W. Hauser and H. Feshbach, Phys. Rev. 87, 366 (1952).

[62] R. Vandenbosch and J. R. Huizenga, Nuclear Fission (Academic, New York, 1973).

[63] H. Stöcker and W. Greiner, Phys. Rep. 137, 277 (1986).

[64] J. Aichelin, Phys. Rep. 202, 233 (1991).

[65] A. Bonasera, F. Gulminelli, J. Molitoris Phys. Rep. 243, 1 (1994).

[66] R.G. Korteling, R.E.L. Green, J.M. D'Auria, R.L. Helmer, K.P. Jackson, S.B. Kaufman and B.D. Wilkins, Phys. Rev. C 41, 2571 (1990).

[67] C. Beck and A. Szanto de Toledo Phys. Rev. C 53, 1989 (1996).

[68] B.D. Wilkins, E.P. Steinberg and R.R.Chasman, Phys. Rev. C 14, 1832 (1976).

[69] C. Böckstiegel, S.Steinhäuser, J.Benlliure, H.-G. Clerc, A. Grewe, A. Heinz, M. de Jong, A.R. 
Junghans, J. Müller and K.-H. Schmidt, Phys. Lett. B398, 259 (1997).

[70] R. Bass, in Proceedings of the Symposium on Deep-Inelastic and Fusion Reactions with Heavy Ions, Berlin 1979 (Springer-Verlag, Berlin 1980).

[71] R. Bass, in Nuclear Reactions with Heavy Ions, (Springer-Verlag, Berlin 1980).

[72] P. Napolitani, GSI annual report, NQMA-EXP-01, 117 (2004).

[73] D. Henzlova, arXiv: nucl-ex/0801.3110v1.

[74] S.J. Sanders, D.G. Kovar, B.B. Back, C. Beck, D.J. Henderson, R.V.F. Janssens, T.F. Wang, and B.D. Wilkins, Phys. Rev. C 40, 2091 (1989).

[75] C. Beck, B. Djerroud, F. Haas, R.M. Freeman, A. Hachem, B. Heusch, A. Morsad, M. Youlal, Y. Abe, A. Dayras, J.P. Wieleczko, B.T. Matsuse, S.M. Lee Zeitschrift fr Physik A Hadrons and Nuclei 343, 309 (1992).

[76] Sl. Cavallaro, E. De Filippo, G. Lanzanò, A. Pagano, M.L. Sperduto, R. Dayras, R. Legrain, E. Pollacco, C. Beck, B. Djerroud, R.M. Freeman, F. Haas, A. Hachem, B. Heusch, D. Mahboub, A. Morsad, and R. Nouicer Phys. Rev. C 57, 731 (1998).

[77] L.B. Yang, E. Norbeck, W.A. Friedman, O. Bjarki, F.D. Ingram, R.A. Lacey, D.J. Magestro, M.L. Miller, A. Nadasen, R. Pak, N.T.B. Stone, A.M. Vander Molen, G.D. Westfall, S.J. Yennello, Phys. Rev. C 60, 041602(R) (1999)).

[78] E.M. Winchester, J.A. Winger, R. Laforest, E. Martin, E. Ramakrishnan, D.J. Rowland, A. Ruangma, S.J. Yennello, G.D. Westfall, A. Vander Molen, E. Norbeck, Phys. Rev. C 63, 014601 (2001)).

[79] I. Lombardo, C. Agodi, F. Amorini, A. Anzalone, L. Auditore, I. Berceanu, G. Cardella, S. Cavallaro, M.B. Chatterjee, E. De Filippo, E. Geraci, G. Giuliani, L. Grassi, J. Han1, E. La Guidara, D. Loria, G. Lanzalone, C. Maiolino, A. Pagano, M. Papa, S. Pirrone, G. Politi, F. Porto, F. Rizzo, P. Russotto, A. Trifiro, M. Trimarchi, G. Verde, and M. Vigilante Phys. Rev. C 84, 024613 (2011)).

[80] M.V. Ricciardi, A.V. Ignatyuk, A. Kelic, P. Napolitani, F. Rejmund, K.-H. Schmidt and O. Yordanov, Nucl. Phys. A733, 299 (2004).

[81] P.Napolitani, F.Rejmund, L.Tassan-Got, M.V.Ricciardi, A.Kelic, K.-H.Schmidt, O.Yordanov, A.V.Ignatyuk and C.Villagrasa, Int. J. Mod. Phys. E13, 333 (2004).

[82] B.L. Tracy, J. Chaumont, R. Klapisch, J.M. Nitschke, A.M. Poskanzer, E. Roeckl and C. Thibault, Phys. Rev. C 5, 222 (1972).

[83] T. Matsuse, C. Beck, R. Nouicer and D. Mahboub Phys. Rev. C 55, 1380 (1997).

[84] C. Beck, Nucl. Phys. A738, 24 (2004).

[85] W. von Oertzen, Eur. Phys. J. A, 29, 133, (2006).

[86] Lecture Notes In Physics 818, Clusters in Nuclei, Edt: C. Beck, Springer, Vol.1 (2010), Vol.2 (in press)

[87] A. Schüttauf, W.D. Kunze, A. Wörner, M. Begemann-Blaich, Th. Blaich, D.R. Bowman, R.J. Charity, A. Cosmo, A. Ferrero, C. K. Gelbke, C. Gross, W.C. Hsi, J. Hubele, G. Immé, I. Iori, J. Kempter, P. Kreutz, G.J. Kunde, V. Lindenstruth, M.A. Lisa, W.G. Lynch, U. Lynen, M. Mang, T. Möhlenkamp, A. Moroni, W.F.J. Müller, M. Neumann, B. Ocker, C.A. Ogilvie, G.F. Peaslee, j. Pochodzalla, G. Raciti, F. Rosenberger, Th. Rubehn, H. Sann, C. Schwarz, W. Seidel, V. Serfling, L.G. Sobotka, J. Stroth, L. Stuttge, S. Tomasevic, W. Trautmann, A. Trzcinski, M.B. Tsang, A. Tucholski, G. Verde, C.W. Williams, E. Zude, B. Zwieglinski Nucl. Phys. A607, 457 (1996).

[88] M. V. Ricciardi, T. Enqvist, J. Pereira, J. Benlliure, M. Bernas, E. Casarejos, V. Henzl, A. Kelic, J. Taieb, K.-H. Schmidt, Phys. Rev. Lett. 90, 212302, (2003).

[89] L. Beaulieu, T. Lefort, K. Kwiatkowski, R.T. de Souza, W.-c. Hsi, L. Pienkowski, B. Back, D. S. Bracken, H. Breuer, E. Cornell, F. Gimeno-Nogues, D.S. Ginger, S. Gushue, R.G. Korteling, R. Laforest, E. Martin, K. B. Morley, E. Ramakrishnan, L.P. Remsberg, D. Rowland, A. Ruangma, V.E. Viola, G. Wang, E. Winchester, and S.J. Yennello, Phys. Rev. Lett. 84, 5971, (2000). 